\title{
The Promoting Effects of Activated Olfactory Ensheathing Cells on Angiogenesis after Spinal Cord Injury Through the PI3K/Akt Pathway
}

Xiaohui Wang

Xi'an Jiaotong University

Chao Jiang

Xi'an Jiaotong University

Yongyuan Zhang

Xi'an Jiaotong University

Zhe Chen

Xi'an Jiaotong University

Hong Fan

Xi'an Jiaotong University

Yuyang Zhang

Xi'an Jiaotong University

Zhiyuan Wang

Xi'an Jiaotong University

Fang Tian

Xi'an Jiaotong University

Jing Li

Xi'an Jiaotong University

Hao Yang

Xi'an Jiaotong University

Dingjun Hao ( $\nabla$ haodingjun@mail.xjtu.edu.cn )

Xi'an Jiaotong University https://orcid.org/0000-0002-1405-8065

\section{Research}

Keywords: Olfactory ensheathing cells, spinal cord injury, angiogenesis, cell transplantation, endothelial cells, PI3K/Akt

Posted Date: January 7th, 2022

DOI: https://doi.org/10.21203/rs.3.rs-1208796/v1 
License: (c) (i) This work is licensed under a Creative Commons Attribution 4.0 International License. Read Full License

Version of Record: A version of this preprint was published at Cell \&amp; Bioscience on March 4th, 2022. See the published version at https://doi.org/10.1186/s13578-022-00765-y. 


\section{Abstract}

Objective

The aim of this study was to investigate the pro-angiogenic potential of olfactory ensheathing cells (OECs) activated by curcumin (CCM) and lipopolysaccharide (LPS) and the possible underlying mechanisms.

Methods

Vascular endothelial cells or tissues were cultured and treated with conditioned medium (CM) extracted from the activated through the addition of LPS and CCM or unactivated OECs. Concomitantly, the proangiogenic potential of OECs was assessed in vitro by aortic ring sprouting assay, endothelial wound healing assay, CCK-8 assay and tube formation assay. Subsequently, the OECs were co-cultured with endothelial cells to evaluate their promoting effect on the proliferation and migration of endothelial cells following undergoing a mechanical scratch. Moreover, the spinal cord injury (SCI) model in rats was established, and the number of endothelial cells and vascular structure in the injured area after $\mathrm{SCl}$ was observed with OECs transplantation. Finally, the underlying mechanism was investigated by western blot analysis of phosphorylated kinase expression with or without the MK-2206 (Akt-inhibitor).

Result

The present results showed that the activated OECs can effectively promote the proliferation, migration and vessel-like structure formation of vascular endothelial cells. Strikingly, several pro-angiogenic growth factors such as VEGF-A and PDGF-AA, which facilitate vessel formation, were found to be significantly elevated in CM. In addition, the PI3K/Akt signaling pathway involved in pro-angiogenic event caused by activated OEC CM, displaying higher phosphorylation levels in cells. On contrary, the delivery of MK2206 can effectively abrogate all the positive effects.

\section{Conclusions}

OECs activated by LPS and CCM, have a strong pro-angiogenesis effect, and can effectively promote angiogenesis and improve the injury microenvironment when transplanted in injured spinal cord. This potentiated ability of OECs to pro-angiogenesis is likely mediated through the PI3K/Akt pathway.

\section{Introduction}

Spinal cord injury (SCl) can cause a permanent loss or reduction in motor and sensory functions below the damage level which following axonal and cellular damage, along with ischemic changes and inflammatory infiltrates. $[1,2]$ Cells are complex systems with multiple targets and stimuli-responsive functions, therefore, cells from several different tissue sources have great potential to treat patients with $\mathrm{SCl}$, cell therapy can improve $\mathrm{SCl}$ regeneration from multiple targets, including but not limited to immune 
regulation, nutritional support, neuronal replace and axon reconstruction. [3] These characteristics fit well with foregoing complex pathological changes of $\mathrm{SCl}$ as their therapeutic targets.

Olfactory ensheathing cells (OECs) are suitable to achieve neuroplasticity/neuroregeneration in SCI so this kind of cells have been intensively studied as potential candidates for cell transplant therapy of SCl. $[4,5]$ These studies mainly explore its function or strengthen its function. Several bioactive factors derived from OECs improved the microenvironment of the injured areas indirectly, and in term of directly contact, OECs were considered to have crosstalk with astrocytes and glial scars which can limit and reconstruct glial scars. Besides, their phagocytosis is beneficial too. $[4,6-10]$ These functions jointly promote inflammatory regulation, neurotrophic and axonal induction of injured area which can provide a microenvironmental basis for nerve repair. However, due to the different time and induction environment of cell transplantation, it will affect the function of OECs. [11, 12] To further maintain the functional stability and develop the potential of OECs, optimization of the sampling method and source of OECs, or beneficial pretreatment of cultured OECs before transplantation, will greatly enhance the ability of OECs to regulate the microenvironment in spinal cord injury. $[13,14]$ For example, we recently observed that OECs can acquire better bioactivity by the way of pretreated by lipopolysaccharide (LPS) and/or curcumin (CCM), such as phagocytic capacity and promotive effects on neuronal growth. [14-16]

For example, we recently observed that OECs can acquire better bioactivity by the way of pretreated by lipopolysaccharide (LPS) and/or curcumin (CCM), such as phagocytic capacity and promotive effects on neuronal growth.

The maintenance of blood supply is an important basis of microenvironmental homeostasis, whereas primary mechanical damage from SCl leads to the disruption of the topical capillaries and the bloodbrain-spinal cord barrier (BSCB), and then ischemic changes occur in the spinal cord due to inflammation, edema, and vascular endothelial cells damage. [17] Under the continuing effects of these adverse changes and other secondary injury, the new functional vessels in and near the injury epicenter unable to formation sufficiently. [18] Both axon regeneration and inflammatory product removal are dependent on the vascular system, so there is no doubt that ischemia worsens the microenvironment of the injured area and increases the difficulty of neurological recovery. $[19,20]$ Therefore, the promotion of blood-supply regeneration should not be neglected in exploring the conditions of nerve recovery after SCl. Furthermore, amelioration of angiogenesis should even be considered as the main strategy for early intervention. [2123] After all, the regeneration of vascular system is the structural scaffold and nutritional basis for nerve regeneration.

Although there are many studies on the effect of OECs transplantation on the improvement of microenvironment and nerve recovery after $\mathrm{SCl}$, few literatures have reported the effects of OECs on vascular endothelial cells or spinal vascular system. But what makes sense is that OECs can express vascular endothelial growth factor (VEGF) was confirmed by related studies, which is considered to be the most effective growth factor in promoting angiogenesis. [24-26] These studies have preliminarily 
revealed the potential of OECs to promote angiogenesis, and this is exactly what is essential after vascular system injury of spinal cord.

Earlier, both Richter and Ramer's research groups found the phenomenon of increased vascular structure and directional angiogenesis in animal models of $\mathrm{SCl}$ treated by OECs transplantation (Staining: RECA for vascular endothelial cells). $[27,28]$ However, due to the complexity of the in vivo environment and their research orientation, they did not systematically describe the behavioral changes and related mechanisms of angiogenic stimulation of OECs. Overall, the role of OECs in angiogenesis is still neglected compared with other studies for microenvironmental changes.

In our study, we systematically observed the changes of vascular endothelial cells and vascular tissues after OECs intervention in vivo and in vitro, involving indirect effects (conditioned media for OECs) and direct effects (co-culture in vitro and cell transplantation in vivo). And furthermore, based on the results of growth factor array screening in conditioned medium, we verified the possible molecular pathways and discussed the mechanism behind the phenomenon. In terms of design, on the basis of conventional isolation and culture of OECs, we also used the OECs pretreated with LPS and CCM reported previously as the activated group to explore whether the pretreated OECs had better effects. $[15,16]$

\section{Materials And Methods}

\section{Main reagents}

Dulbecco's modified Eagle's medium (DMEM)/F12, fetal bovine serum (FBS), 0.25\% EDTA-trypsin, DMEM and penicillin/streptomycin solution were purchased from Gibco (Carlsbad, CA, USA); LPS, CCM, polyLysine (PLL), dimethyl sulfoxide (DMSO), bovine serum albumin (BSA), $0.2 \%$ gelatin and crystal violet dye were purchased from Sigma-Aldrich (St. Louis, MO, USA); Dispase (neutral protease, grade II) was from Roche (Basel, Switzerland); Anti-p75 antibody, anti-CD31 antibody, anti-pan-Akt antibody, anti- PI3K p85 a antibody, anti phospho-PI3K p85a (Y607) antibody and 5-ethynyl-2-deoxyuridine (EdU) Proliferation Kit (iFluor 488) were purchased from Abcam (Cambridge, MA, USA); Anti-phospho-Akt (Ser473) antibodies were from Cell Signaling (Danvers, MA, USA). Human umbilical vein endothelial cells (hUVECs), endothelial cell medium (ECM) and endothelial cell growth supplement (ECGS) were purchased from Sciencell (San Diego, CA, USA); Red Fluorescent Probe M02 kit was purchased from Bestbio (Shanghai, China); MK-2206 was from Selleck Chemicals (Houston, TX, USA); Cell counting kit-8 (CCK-8) proliferation assay, western blotting kit and anti- $\beta$-actin antibody was purchased from Boster (Wuhan, Hubei, China); DAPI Kit, Fluor594-conjugated goat anti-rabbit IgG and Fluor594-conjugated goat anti-mouse were purchased from Molecular Probes (Eugene, OR, USA). Anti-mouse/rabbit immunohistochemistry detection kit was from Proteintech (Cambridge, MA, USA). MICROFIL ${ }^{\circledR}$ angiography kit was purchased from Flowtech (Carver, MA, USA). $35 \mathrm{~mm}$ dishes, cell culture plates, plastic coverslips and flasks were all purchased from Thermo Fisher (Shanghai, China). Matrigel basement membrane matrix and 24-well Transwell plates were purchased from Corning (Corning, NY, USA); $35 \mathrm{~mm}$ confocal dishes and centrifuge 
tubes were from NEST (Wuxi, Jiangsu, China). Microcentrifuge tubes (EP tubes) were purchased from Eppendorf (Hamburg, German).

\section{Primary Culture, Purification and Identification of OECs}

All experimental protocols involving experimental animals used in this study were reviewed and approved by the ethics committee at Honghui Hospital, affiliated with Xi'an Jiaotong University and conformed to the Guide for the Care and Use of Laboratory Animals published by the National Institutes of Health. As described in the previous literature, the OECs were isolated and primarily cultured from olfactory blubs in rats at 2-3 months (Fig. s1), and further purified by the modified differential adherent velocity method of $\mathrm{H}$. Nash et al. $[15,29]$ There are also minor modifications that we removed tractus olfactorius (inner layer) to the maximum extent, and retained the outer olfactory bulb layer (nerve layer and glomerulus layer), which is based on the distribution characteristics of OECs, in order to eliminate the interference of other glial cells. [13] After purified, the OECs were reseeded onto 25-cm² PLL-coated culture flasks and maintained in DMEM/F12 media with $10 \% \mathrm{FBS}$, cultured at $37^{\circ} \mathrm{C}$ and $5 \% \mathrm{CO}_{2}$ in an incubator (Thermo Fisher, Waltham, MA, US). Half the media were changed every 3 days. Usually around 10 days after isolated, the confluence of OECs reached more than $80 \%$, the OECs can then be identified by immunofluorescence staining or activated by CCM\&LPS. When we prepared OECs for cell co-culture in vitro and transplantation in vivo, enhanced green fluorescent protein (GFP) transgenic rats were selected as the olfactory bulb donor to observe the distribution of OECs more intuitively, all other methods were the same.

\section{Activation of OECs and Conditional-media (CM) Collection}

When the OECs reached the confluence conditions that can be treated, cells were digested by $0.25 \%$ EDTA-trypsin and made into suspensions, divided equally into two groups and then reseeded on 6-well plates (for experimental operation) or coverslips (for immunofluorescence identification). One group was noted as Activated-OECs (Act-OECs), the media of OECs in this group were treated with $1 \mu \mathrm{g} / \mathrm{ml}$ LPS and $1 \mu \mathrm{M}$ CCM previously described by Hao et al. The other group was noted as Unactivated-OECs (UnaOECs), its media just were added equal amounts of DMSO (solvent for CCM and LPS). To investigate whether the OECs were able to promote angiogenesis of vascular endothelial cell, we collected the conditioned media of OECs from the aforementioned cell culture, which were noted as ACM and UCM, respectively, and DMEM/F12 media with 10\%FBS were used as no-treatment control group. After treated one day, media mixed with drugs or DMSO were removed, and completely replaced as complete media. To ensure adequate nutrients, conditioned media were collected once every day from the next day, a total of three times. Three days later, these OECs supernatants were combined, mixed, and centrifuged at $14,000 \times \mathrm{g}$ for $15 \mathrm{~min}$ at $4^{\circ} \mathrm{C}$ and then filtered with a sterile $0.22-\mu \mathrm{m}$ pore to remove cell debris. Finally, these conditioned media were kept at $-80^{\circ} \mathrm{C}$ until used.

\section{Rat Aortic Ring Assay (Neovascularization)}


This operation is performed based on the previous description. [30,31] Rat aortic rings were isolated from young rats (three-week-old, weighing $50 \mathrm{~g} \pm 10 \mathrm{~g}$ ), and incubated in serum free DMEM overnight as starved pretreatment. Before the aortic ring sprouting assay, $100 \mu \mathrm{L}$ of the Matrigel was added to each well of a 96-well plate by precooled pipette tip, one aortic ring per well was embedded in the middle of the gel. Then plates were moved in a $37^{\circ} \mathrm{C}$ cell incubator for half an hour for gel to set. (Fig. s2b) And then add $150 \mu \mathrm{L}$ ACM or UCM or control media with $10 \%$ FBS to each well by group set, each group had three replicates. These aortic rings were cultured at $37^{\circ} \mathrm{C}$ and $5 \% \mathrm{CO}_{2}$ for 7 days, and then the sprouting results were photographed under an inverted phase contrast microscopy, Leica DM IL (Leica Microsystems, Germany). Each test process was repeated three times and relevant sprouting indexes were calculated digitally using Image J software (National Institutes of Health, Rockville, MD, USA).

\section{Endothelial Wound Healing (Cell Migration) Assay}

Migration of endothelial cells was determined using a two-dimensional wound-scratch assay on 24-well plates. HUVECs were seeded onto each well of 24 -well plates $\left(1 \times 10^{5}\right.$ cells/well) and maintained in DMEM with $10 \%$ FBS for $24 \mathrm{~h}$, followed by overnight starvation with serum free media. At the bottom of each hole, adherent cells were scratched by a $10 \mu \mathrm{L}$ pipette tips radially through the center, and then each hole was washed with PBS (phosphate buffer solution) three times to remove the floating cells. The wound can be seen under a microscope immediately. Next, the hUVECs were cultured in $500 \mu \mathrm{L}$ ACM or UCM or Control media by group set. To really examine the motility contribution to the healing and to exclude the component related to cell proliferation, hUVECs are incubated with the antimitotic agent mitomycin (Sigma-Aldrich, USA). The wounds were observed using an inverted phase contrast microscope after 24 hours. Photographs were taken at regular intervals over the course of 24 hours until wound closure was achieved. The operation was repeated three times and the results were analyzed with Image J. Healing index $=($ initial area - final area $) /$ initial area $\times 100 \%$.

\section{CCK-8 Proliferation Assay}

The cultured hUVECs were digested and centrifuged, then the liquid supernatant was removed. Dispense $100 \mu \mathrm{L}$ of experimental group or control group media above-mentioned, after dispersed uniformly, the hUVECs suspension was distributed and inoculated to each well (5000 cells/well) in a 96-well plate. Preincubate the plate for 1 hours in an incubator $\left(37^{\circ} \mathrm{C}, 5 \% \mathrm{CO}_{2}\right)$ until cells were completely adherent. Add 10 $\mu \mathrm{L}$ of CCK-8 solution to each well of the plate. Then incubate the plate for 6 hours in the incubator. During incubation, measure the absorbance at $450 \mathrm{~nm}$ using a microplate reader (Thermo Fisher, Waltham, MA, US) every hour, the $\mathrm{OD}_{450}$ values were recorded.

\section{HUVECs Tube Formation Assay}

Matrigel was coated in each well of a precooled 96 -well plate $\left(50 \mu \mathrm{L} /\right.$ well), plates at $37^{\circ} \mathrm{C}$ for $0.5 \mathrm{~h}$. Then, $3 \times 10^{4}$ HUVECs were seeded with the media of preset groups on Matrigel as Fig. s2c, the method of cell suspension by medium is previously described. The tube formation ability of HUVECs was measured at 3 , 6, 9 h. After incubation, images were captured by an inverted phase contrast microscope and the capillary 
length and the number of nodes/junctions/meshes of the tubular structures was quantified by Image $\mathrm{J}$ and repeated these processes three times independently.

\section{Isolating and Primary culture of Rat Aortic Endothelial Cells (RAECs)}

The aorta of young rats (three-week-old, weighing $50 \mathrm{~g} \pm 10 \mathrm{~g}$ ) was isolated and obtained under aseptic conditions. Under an anatomical microscope (OPTON, German), the clot and connective tissue are stripped from the aorta using ophthalmic microscopic surgical instruments. After washed by DMEM with $1 \%$ penicillin/streptomycin three times, a surgical suture was threaded through the active vessels and the ends of the vessels are ligated. (Fig. s3a-e) Then the intima of aortas was turned over and exposed by suture-pulling, the unligated end is closed by a vascular clamp. (Fig. s3f) The aorta was placed in $1 \mathrm{mg} / \mathrm{ml}$ Dispase in an incubator for 60 min to digest the collagen in the cell matrix in this inverted state of the intima. Next, the aorta was transfer to DMEM with 10\% FBS and dissected into small slices about 2 $\mathrm{mm} \times 2 \mathrm{~mm}$. These slices are laid down at the bottom of the gelatin-coated 6-well plate (make sure that the intima faces downward), 1-2 per well, as shown in Fig. s3g. At this time, the slices were not adherent to the plate, so the medium should be maintained to just moisten the bottom of the slices without flooding it, the amount of ECM with 5\% FBS and 1\% EGS added at each well is about $800 \mu \mathrm{L}$. After that, the 6-well plates were placed in a small wet box and cultured at $37^{\circ} \mathrm{C}, 5 \% \mathrm{CO}_{2}$, media were changed every 48 h. At 4-5 days after incubation, RAECs can be seen crawling out from the tissue margins under a microscope, at this time, the tissue slices can be removed and then added $2 \mathrm{~mL}$ media to each well of plates, media should be changed every other day. After day 10, the RAECs could be subcultured, cryopreserved or used for further experiments. In addition, these cells were identified by immunofluorescence using anti-CD31 antibody.

\section{Co-culture of OECs and RAECs in vitro}

After primary cultures, collection many times and mixing, the RAECs were reseed at each well of gelatincoated 24-well plates $\left(1 \times 10^{5}\right.$ cells/well) under DMEM/F12 with 10\% FBS for 2 hours to let cells adapt to the new environment. Then the original medium was then replaced with the medium containing $0.5 \%$ red fluorescent probe M02. Two hours later, the cells were labeled with fluorescence, then washed with PBS for three times and replaced with a medium without probe. Similar to wound healing - cells migration assay, the wounds were generated by manually scratching the cell surface with $10 \mu \mathrm{L}$ pipette tips, which can mimic the conditions of vascular endothelial injury in vivo. After that, suspension of pre-digested activated OECs or unactivated OECs were added into the holes of different groups respectively (Medium: F12/DMEM +10\% FBS). Medium without cells was set as no-treatment control. All steps were repeated for three times. Refer to previous results, [32] before and after scratch, co-culture $1 \mathrm{~h}$ and co-culture $24 \mathrm{~h}$ were set to the shooting time, and the wound healing was analyzed by the merge images involved red fluorescence (M02: RAECs), green fluorescence (GFP: OECs) and bright field (all cells). The healing index was calculated as described above.

\section{Animal and Experimental Setting}


Forty-eight female Sprague-Dawley rats weighing $200 \pm 30 \mathrm{~g}$ (specific pathogen free) were obtained from the Laboratory Animal Centre of Xi'an Jiaotong University. The rats were housed at constant temperature $\left(23 \pm 2^{\circ} \mathrm{C}\right)$ and humidity of $50 \% \pm 10 \%$ on a $12 / 12 \mathrm{~h} \mathrm{light/dark}$ cycle with constant air renewal. The animals were divided into four groups at random as fellows: (i) in the sham group, rats were subjected to laminectomy but not $\mathrm{SCl}$; (ii) in the $\mathrm{SCl}$ group, rats were subjected to laminectomy and compressional $\mathrm{SCl}$; (iii) in the activated-OECs transplantation group (AOT), concentrated suspension of activated OECs was transplanted on the basis of SCl group; (iv) in the unactivated-OECs transplantation group (UOT), concentrated suspension of unactivated OECs was transplanted on the basis of SCl group. Each group contained 12 experimental animals, behavior and morphology were observed after operation.

\section{Animal SCI model and OECs Therapy}

20 minutes before surgery, rats in each group were induced anesthesia by isoflurane with veterinary anesthesia machine (RWD, Shenzhen, Guangdong, China), afterwards rats were anesthetized intraperitoneally with $1 \%$ sodium phenobarbital $(4 \mathrm{~mL} / \mathrm{kg})$. Concomitantly, the rats were immobilized in the prone position. Vaseline oil was used on the eyes to prevent drying during surgery. After hair removal, the operational locations were disinfected with medical iodine volts. The operational method for compressional SCI model were mainly performed as previously described [33] Briefly, for one case, a longitudinal incision was performed overlying the T7-11 area using operating scissors under aseptic conditions. After subperiosteal of paraspinal muscles, a laminectomy was performed to expose its spinal cord from T9 or T10 (Fig. s4a). In the exposed area of its spinal cord, a compressional lesion was produced using a calibrated compression method with microsurgical forceps (Yunkang, Jiangsu, China) for $20 \mathrm{~s}$, then remove the forceps carefully [34] the tip spacing of forceps is $1 \mathrm{~mm}$ during compression. (Fig. s4a-c)

OECs transplantation was performed with the rats positioned in a stereotaxic instrument (RWD). Either 5 $\mu \mathrm{L}$ of a cell suspension containing $1 \times 10^{5}$ cells or saline alone ( $\mathrm{SCl}$ group) was injected into the core site of the injured spinal cord by a $10 \mu \mathrm{L}$ siliconized Hamilton syringe with a beveled glass pipette tip $(80 \times 90$ $\mathrm{mm}$ inner diameter) (Fig. 4a). Injection speed is uniform and slow $(0.2 \mu \mathrm{l} / \mathrm{min})$ and the glass pipette was left in position for an additional 5 min to prevent leakage before withdrawal, the whole cell transplant process takes half an hour. [15]

After that, using sterile normal saline to washout the incisal opening, sewing up each layer tissues in order. Three days after the operation, the rats were fasted but add appropriate amount of glucose to water for energy, and given daily intraperitoneal injection of cefuroxime to prevent infection. Besides, urination can be artificially assisted by massaging the bladders every day. Gradually adjust the feed quantity according to their behavior recovery.

\section{Basso, Beattie, and Bresnahan (BBB) Locomotor Scale}

The establishment effect of model and treatment effect were evaluated by the BBB locomotor scale by the methods described in original literature. [35] The scores for each group were recorded $1 d, 3 d, 7 d, 14$ $d$, and $21 d$ post-injury. The BBB scores for each group were observed and recorded by two trainees who 
were not aware of the group allocation, take the average and round it. The scoring criteria included joint movement, paw placement, and coordination, these data of animals were recorded by two trainees in a noise-free, open field arena for $5 \mathrm{~min}$ at least. The rats with normal motor function obtained a BBB score of 21 points, while the rats losing motor function completely were scored 0 point. Finally, data compiled were analyzed using two-sample t-test and linear mixed effects model analysis.

\section{Immunofluorescence and Immunohistochemistry}

OECs and RAECs on plastic coverslips of all groups were fixed with $4 \%$ paraformaldehyde (Sigma), for 30 min, and then treated with $3 \%$ BSA in $0.01 \mathrm{M}$ PBS as nonspecific blocker for $30 \mathrm{~min}$. Coverslips were incubated with primary rabbit monoclonal antibodies against p75 (1:500, for OECs) or mouse monoclonal antibodies against CD31 (1:1000, for RAECs), at $4^{\circ} \mathrm{C}$ overnight, washed in PBS three times and incubated with the corresponding fluorescence conjugated secondary antibody (Alexa Fluorß 594 goat anti-rabbit IgG and Alexa Fluor ${ }^{\circledR} 594$ goat anti-mouse $\lg \mathrm{G}$ antibody, 1:1000 dilution) for $2 \mathrm{~h}$ and DAPI nuclear staining solution (1:1000) at room temperature(RT) for $10 \mathrm{~min}$ in order, then invert the coverslips onto the glass slides sealed by antifading mounting medium (Boster) after rinsed in PBS. All cells were primarily cultured independently for three times at least.

For animal models at 7-day post-surgery, after be anesthetized with $1 \%$ sodium phenobarbital $(4 \mathrm{~mL} / \mathrm{kg})$, their left ventricle was poured into saline and perfusion fixed by $4 \%$ paraformaldehyde. Their spinal cords were isolated from the vertebral canal and post-fixed in $4 \%$ paraformaldehyde at $4^{\circ} \mathrm{C}$ for three days, cryoprotected in $30 \%$ sucrose in 0.1 M PBS for 1 week, and cut into segments, embedded in optimum cutting temperature compound (SAKURA Tissue-Tek, CA, USA) and sectioned at $10 \mathrm{~mm}$ thickness and pasted into PLL coated slides for immunofluorescent and histochemical staining analysis.

Immunofluorescence of histological sections were performed following previously described methods of immunofluorescence staining of cells, but the additional step is that the sections were incubated with $0.01 \%$ triton-X100 (Sigma-Aldrich, USA) for $1 \mathrm{~h}$ at RT before BSA incubation.

One week after injury, CD31-positive vascular endothelial cells were identified and quantified at the spinal lesion site by immunohistochemistry method. Briefly, at RT, the histological sections were incubated with $3 \% \mathrm{H}_{2} \mathrm{O}_{2}$ for 10 min to inactivate the endogenous enzymes firstly, and then incubated in BSA for 1 hour, incubated overnight at $4^{\circ} \mathrm{C}$ with anti-CD31 antibody, washed three times by PBS. After it was combined with Goat anti-Mouse/Rabbit Poly- horseradish peroxidase (HRP) secondary antibody, 3,3' Diaminobenzidine Tetrahydrochloride was added to the tissue to produce chromogenic reaction. After washing, it was redyed with hematoxylin for 2-3 minutes, then rinsed with distilled water, followed by gradient dehydration with alcohol $(60 \%, 75 \%, 100 \%)$ for 5 minutes each grade. After removal, it was placed in xylene twice for 5 minutes, sealed with neutral balsam (Bestbio, China) and observed at last.

All slides were observed under an of a Leica DM6 B microscope (Leica Microsystems, Germany). All images were captured using the Leica LAS X software (Leica Microsystems, Diegem, Belgium). Results were measured by Image $\mathrm{J}$ software. 


\section{Angiography of Spinal Cord}

Paraformaldehyde perfusion fixation was performed on animals in the same way as before, and after that, the circulatory systems of rats were perfused with the Microfil ${ }^{\circledR}$ MV-122 (Yellow) silicone rubber contrast agents. A sign of successful infusion of contrast agent is yellow-dying of the microvessels in the sclera or liver of the animal. After the infusion, the dead animals were stored at $4^{\circ} \mathrm{C}$ to promote the Microfil in the blood vessels reach the full polymerisation. The spinal cords were extracted and cut to remain $1.5 \mathrm{~cm}$ (centered around the lesion). (Fig. s5)

micro-computed tomography (micro-CT) images were obtained using eXplore Locus SP system (General Electric, Milwaukee, WI, USA), the resolution is 5 microns. The angiographic images were reconstructed to 3D vascular models and analyzed by VGStudio MAX (Volume Graphics, Heidelberg, Germany).

\section{Growth Factor Assay}

The growth factors in the CM of OECs were semi-quantitatively evaluated using a multiplex growth factor array system (Rat Growth Factor Array 1; RayBiotech, Norcross, GA, USA). The CM were not required to dilute but calibrated according to cell numbers when the $\mathrm{CM}$ was collected. All processes were operated according to the protocol of user manual. The chemiluminescence signal of each membrane dot was imaged using a ChemiDoc XRS (Bio-Rad, Hercules, CA, USA) and quantified using Quantity One software (Bio-Rad), which allowed for the assessment of growth factor content in the CM. Tests were repeated three times, the CM came from different OECs-culture batches each time. Results were measured by Image J software.

\section{Western Blots}

Based on the result of growth factor assay, for investigation of the signaling molecules involved in mediating the angiogenesis by activated OECs, intervention was performed on three groups that had been set up for in vitro experiments. In each group, MK2206 was dissolved into a solution by DMSO and added into media to achieve a final concentration of $5 \mu \mathrm{M}$. Three groups with MK2206 and three groups without MK 2206 (add equal amount of DMSO) were used as the media for hUVECs culturing respectively. HUVECs were mixed with grouping media to suspensions and inoculated into wells corresponds to the group of 6-well plates ( $10^{5}$ cells/ well). (Fig. s6d) After $24 \mathrm{~h}$ of culture, to analyze changes in phosphorylation of Akt and PI3K, total cellular extracts were prepared as follows: hUVECs in six wells were carefully but briefly rinsed with saline buffer and extracted in ice-cold RIPA as described by Yang et al. [36] It is noteworthy that phenylmethanesulfonyl fluoride and phosphatase inhibitor (Beyotime, Jiangsu, China) is added into the PIPA (1:50) to prevent protein degradation and dephosphorylation. [37] The protein concentrations of clarified lysates were measured using the BCA protein assay (Qiagen, Germany), with BSA as a reference. The following antibodies were used: pan-Akt (1:500), phospho-Akt (1:2000), PI3K p85 a (1:1000), and Phospho-PI3K p85a (1:500). All protein lysates were run on 10\% gradient SDS-PAGE for $1.5 \mathrm{~h}$, transferred onto poly vinylidene fluoride (PVDF) membranes for 50 min and blocked with $5 \%$ fat-free milk for $1 \mathrm{~h}$ at RT for 30 min, washed with Tris-buffered saline-Tween 20 (TBST) 
and probed with four kinds of primary antibodies at $4^{\circ} \mathrm{C}$ overnight. $\beta$-actin was included as an internal loading control. After washed, the membrane was incubated with HRP-conjugated secondary antibody at RT for $1 \mathrm{~h}$ and washed again with TBST. The immunocomplex bands at PVDF membranes were detected using a ChemiDoc XRS (Bio-Rad, Hercules, CA, USA) and all western blotting experiments were repeated three times. The intensity of density value (IDV) was analyzed using ImageJ software.

\section{Cell Transwell Migration Assay}

For evaluating PI3K/Akt pathway involved in mediating the endothelial cell migration by activated OECs, the chemotactic motility of hUVECs was determined using Transwell migration chambers with $6.5 \mathrm{~mm}$ diameter polycarbonate filters (8- $\mu \mathrm{m}$ pore size). As shown in Fig. $s 2 \mathrm{~d}$, the lower chambers were filled with $600 \mu \mathrm{L}$ of grouping media containing $5 \mu \mathrm{M}$ MK 2206 or DMSO. hUVECs $\left(3 \times 10^{4} /\right.$ well) were seeded in upper chambers in $100 \mu \mathrm{L}$ serum-free DMEM. Cells were allowed to migrate for $8 \mathrm{~h}$. Non-migrated cells were removed with cotton swabs, and migrated cells were fixed with ice cold methanol and stained with $0.1 \%$ crystal violet. Cells were inspected at inverted light microscopy (20x), Leica DM IL. Images were captured by Leica LAS AF Lite software (Leica Microsystems, Germany) and quantified by Image J software.

\section{EdU incorporation assay}

All operations are carried out according to the kit instructions, briefly, hUVECs were seeded in the coverglass bottoms of $35 \mathrm{~mm}$ confocal dishes at a density of $1.5 \times 10^{4}$ cells per dish along with EdU at a working concentration of $10 \mu \mathrm{M}$ in $200 \mu \mathrm{L}$ grouping media for 1 hour. After labeled by EdU, cells were fixed and permeabilized by fixative solution and permeabilization buffer at RT. The mixed reaction solution is prepared by mixing copper sulfate, iFour 488 Azide and TBS, and add the reaction mix into each dish for 30 min at RT, following by washing with PBS and staining with DAPI (1:1000) for 10 minutes at RT. After the staining, the cells were imaged under a fluorescent microscope, Leica DM IL and quantified by Image $\mathrm{J}$ software. All the assays were repeated in three times.

\section{Statistical analysis}

All data are presented as mean \pm standard deviation (SD), and the statistical analyses were undertaken using GraphPad Prism7 software (La Jolla, USA). Statistical significance between groups was determined by analysis of variance (ANOVA). A Student's t-test (normal distribution) was applied to compare the difference between the control and experimental groups. Statistical significance was defined as $P<0.05$.

\section{Results}

\section{Characterization and identification of OECs}

OECs were cultured and observed as described in the method section, ten days after primary culturing, the OECs were taken under invert phase-contrast microcopy before subcultures. The purified OECs have 
formed long, threadlike cellular processes connected to each other in a network (Fig. 1a). Observed under a 20x magnification objective lens, it can be seen that the cell bodies were in a bipolar, multipolar, or an irregular shape (Fig. 1b). Before sub-culture, some OECs were digested and transfer them adherent to plastic coverslips for immunofluorescence identification. Immunocytochemical staining for p75 (a characteristic marker for OECs) demonstrated that over $85 \%$ of cells were OECs (Fig. 1c and f). A bottle of OECs is usually divided equally and one group of OECs is activated randomly as the activated group. After three days of sub-culturing and activation, OECs was observed under phase contrast microscope. It could be observed that both groups of cells had thicker processes than before sub-culturing, and for activated OECs, more cellular body showed bipolar in shape, their processes were also extending parallel directions visually, subjectively, the whole cellular distribution looks tidier (Fig. 1d and e).

\section{Effect of activated OECs-CM on biological behaviors of vascular endothelial cells}

The rat aortic ring assay was been utilized to examine the neovascularization-promoting effect of OECs$\mathrm{CM}$ in vitro, under the same other experimental conditions, the $\mathrm{CM}$ collected from OECs an indeed induce the aortic ring to sprout emerging from the aorta, and with wider and denser network than original complete media $(P<0.05)$. This phenomenon was more significant in of $\mathrm{CM}$ collected from activated OECs (Fig. 2a and b). To confirm OECs-CM induced endothelial migration, we cultured the hUVECs in control media and CM, using an in vitro scratch assay. After $24 \mathrm{~h}$, it can be observed that the scratch areas were refilled by hUVECs, the areas in ACM group were significantly narrowest in three group and UCM group took the second place (Fig. $2 \mathrm{c}$ and d), which indicate that there are some substances promoting the migration of vascular endothelial cells in OECs CM. From our CCK-8 assay results, the percentage of HUVEC proliferation in the ACM and UCM group was obviously increased compared with control media group (ACM vs control: $P<0.01 ;$ ACM $v s$ control: $P<0.05$ ), the ACM group also had the strongest proliferative effect (Fig. 2f). In contrast to the control media and UCM group, longer twodimensional capillary walls and more tubular typical structures (number of nodes, junctions and meshes) were formed on the matrix gel when hUVECs cultured in ACM (Fig. 2e and g-j), this phenomenon of simulating vascular formation is an important experimental basis for carrying out in vivo.

\section{Characterization and identification of RAECs}

The cells began to migrate to the aortic slices $72 \mathrm{~h}$ after inoculation, and grew adherent to the vascular fragments as the center. The morphology was stable after 7 days, the cells cultured on day 9 were observed under an inverted phase contrast microscope, it could be seen that a lot of cells have migrated and adherent (Fig. s3h). The cells showed long spindle shape or polygon shape with bright halo at the edge, who have the characteristics of flat epithelial cells (Fig. 3a and b). Immunocytochemical staining for anti-CD31 antibody (a characteristic marker for vascular endothelial cell) demonstrated that the cells are almost all endothelial cells (Fig. 3c).

Effect of activated OECs on healing of RAECs wound scratches in vitro 
We prepared simulated wound scratches of RAECs in vitro labeled with red fluorescent probe $\mathrm{M} 02$, and then added OECs from GFP transgenic rats to establish a co-culture system (the 1st and 2nd column of Fig. 3d). At $24 \mathrm{~h}$ of co-culture, merged images of fluorescence and bright fields were collected, and it was found that wound scratches in each group had healing to a greater or lesser extent (Fig. 3d). As for the comparison and analysis of the degree of reduction of the area of scratches, the RAECs incubated with activated OECs had the maximum degree of healing, unactivated OECs co-cultured group secured the second place (Fig. 3 e, co-culture of activated OECs vs control: $P<0.05$; co-culture of activated OECs vs coculture of unactivated OECs: $P<0.01)$.

\section{Effect of OECs on angiogenesis after $\mathrm{SCl}$ in vivo}

A portion of the rats were sacrificed one week after $\mathrm{SCl}$, their spinal cord tissue was prepared into $10 \mu \mathrm{m}$ slices and immunofluorescent labeled with anti-p75 antibody. GFP and p75 positive cells almost overlapped, indicating high OECs-purity of transplanted cells. It can be seen from the sagittal view of the spinal cord that OECs all successfully transplanted to the injured area (Fig. 4a). Moreover, activated OECs were more widely distributed than unactivated OECs, and GFP-positive cells extended up and down more. The other portion of the rats ( $n=5$ per group) were observed for 28 days after $\mathrm{SCl}$, their BBB scale were recoded, From the locomotor performance of the control group, the animal model was successfully established, and the recovery of AOT was significantly faster than that of the UOT and SCl group $(P<0.05)$, on the 28th day, the BBB scores of AOT, UOT and SCl group were 13.8 $\pm 1.92,10.40 \pm 1.14$ and $7.80 \pm 1.79$. CD31-positive vascular endothelial cells of rats' spinal cords were labeled by immunohistochemistry. To eliminate the effect of non-specific staining, only the number of strongly positive CD31-positive cells was counted. As shown in Fig. $4 \mathrm{c}$ and d, although the loss of vascular endothelial cells was very severe in spinal cord injuries at week 1 (Sham group vs other group: $P<0.005$ ), more CD31 positive cells were found in the AOT group than in the control group and SCl group yet $(P<0.05)$. In order to presented the three-dimensional structure of spinal blood vessels intuitively, we performed contrast agent perfusion and angiography on each group of animals one week after SCl (Fig. 4e). In terms of percentage of spinal vessel volume (Fig. 4f), there was no significant difference between the AOT group and the sham group one week after $\mathrm{SCl}(P>0.05)$, these two groups were very similar, while the vascular volume of the $\mathrm{SCl}$ and UOT groups was significantly smaller than that of the sham group (Sham group vs UOT group: $P<0.01$; Sham group vs $\mathrm{SCl}$ group: $P<0.05$ ). Mean vessel diameter index and volume index had similar trend (Fig. $4 \mathrm{~g}$ ), the vessel lumen diameter of AOT group was significantly larger than $\mathrm{SCl}$ and UOT groups (both $P<0.05$ ), and it was close to that of SHAM group, but still slightly smaller than that of SHAM group $(P<0.05)$. Also, it is worth noting that the average vessel lumen diameter of the UOT group treat with unactivated OECs-transplantation was $2.41 \pm 0.41$, even slightly smaller than the vessel diameter of the $\mathrm{SCl}$ group with $2.64 \pm 0.33$. In terms of vascular density, both the AOT and UOT groups treated with OECs-transplantation were close to the sham group (both $P>0.05)$, and the difference between the AOT and UOT groups was also small ( $P>0.05)$, with more new blood vessels than the $\mathrm{SCl}$ group, and the vascular density was significantly higher than that of the $\mathrm{SCl}$ group (AOT group vs SCl group: $P<0.001$; UOT group vs SCl group: $P<0.005$ ). 


\section{ACM and UCM contained angiogenesis-related growth factors}

Activated OECs has been shown to promote angiogenesis in vivo and in vitro, further, to account for which angiogenic substances secreted by OECs into the surrounding environment, OECs-CM were screened for angiogenic factors by growth factor array, based on ACM, UCM and control media used in previous experiments in vitro. From Fig. s6a-c and Fig. 5a, the growth factors with relative high enrichment levels (relative intensive ratio in each group $>0.25$ ) and with significantly higher level than the control group $(P<0.05)$ were vascular endothelial growth factor A (VEGF-A), insulin-like growth factorbinding protein-5 (IGFBP-5), platelet derived growth factor AA (PDGF-AA), hepatic growth factor (HGF), glial cell line -derived neurotrophic factor (GDNF), a-nerve growth factor (a-NGF) and brain-derived neurotrophic factor (BDNF). Among the growth factors, the relative enrichment level of VEGF-A PDGF-AA, HGF, GDNF, a-NGF and BDNF in ACM group was significantly higher than that in UCM group. Of these growth factors, VEGF (for vascular endothelial cells) and PDGF (for vascular smooth muscle cells) are thought to have the strongest ability to promote angiogenesis [38, 39].

\section{Activated OECs promote angiogenesis through PI3K/Akt signaling pathway}

Previous studies have demonstrated that the activation of PI3K/Akt pathway can promote the proliferation and migration of vascular endothelial cells and VEGF-A or PDGF -AA can mediate the activation of PI3K/Akt pathway. [40-42] Therefore, combined with our screened growth factors, we hypothesized that there have connections between activated OECs and PI3K/Akt pathway and set extra three Akt-inhibitory groups (MK2206 with Akt inhibitor) on the basis of existing ACM, UCM and control media group. Phosphorylation levels of Akt and PI3K in the lysis of hUVECs cultured in each media-group were evaluated by western blot analysis (Fig. 5b). We found that the levels of phosphorylation in Akt and PI3K of hUVECs from the ACM group were significantly higher than those in the UCM group and control group (Fig. 5c and d). In addition, blotted bands in Fig. 5b and gray-value analysis of Fig. 5c also showed that MK2206 did significantly inhibit the phosphorylation of Akt. These results demonstrated that the phosphorylation level of Akt/PI3K pathway was increased by activated OECs, next we explored whether Akt/PI3K pathway activation could play a role in promoting angiogenesis. Cell motility was assessed with transwell migration assay by inoculated HUVECs in the upper chamber and grouped media in the lower chamber. We observed that the MK2206 supplemented media in the lower chambers induced the less migration of HUVECs through the porous membranes, compared to their corresponding DMSO supplemented media (ACM+DMSO group vs ACM+MK2206 group: $P<0.01, \mathrm{UCM}+\mathrm{DMSO}$ group vs UCM+MK2206 group: $P<0.05$, Control+DMSO group vs Control +MK2206 group: $P<0.01)$. EdU assay was used to analyze hUVECs proliferation under the condition of PI3K/Akt pathway inhibition. The hUVECs cultured in MK2206 supplemented media performed lower EdU incorporation rate than their corresponding DMSO supplemented media (ACM+DMSO group vs ACM+MK2206 group: $P<0.001$, UCM+DMSO group vs UCM+MK2206 group: $P<0.001$, Control+DMSO group vs Control +MK2206 group: 
$P<0.05)$. Besides, similar with the result in CCK-8, it was observed that the hUVECs incubated in ACM had the strongest proliferative activity showed in Fig. $5 \mathrm{~g}$ and h (ACM vs UCM or Control group: $P<0.05)$, whereas the hUVECs cultured in MK2206 supplemented ACM were even significant less proliferative activity than those in MK2206 supplemented UCM (ACM+MK2206 vs UCM+mk2206: $P<0.05)$. In summary, our results suggest that activated OECs can secrete angiogenesis-related growth factors, and promote angiogenesis through the activation of the PI3K/Akt pathway.

\section{Discussion}

The limited repair ability of the neuron and the secondary deterioration of the microenvironment are the main reasons for the difficulty in functional recovery after SCl. [43] Blood supply is closely related to changes in the microenvironment of injured area, primary mechanical trauma from SCl leads to the rupturing and shearing of the microvascular and the blood-brain-spinal cord barrier (BSCB) in the surrounding tissue, which can cause imbalance of hemorrhage and ischemia, further lead to some pathological changes such as inflammatory infiltration and edema of the nerve tissue environment. [44] Intact vascular structure and rich blood supply can promote oxygen/nutrients delivering, and metabolic waste removing, thus the vasculature of the spinal cord plays a crucial role in $\mathrm{SCl}$ and repair, otherwise, tissue repair can be hindered, even deteriorate. $[45,46]$ Massive loss of endothelial cells occur acutely because of impact-generated mechanical forces, during the first $24 \mathrm{~h}$ as a result of necrosis and during the days thereafter mostly as a result of ischemia-induced apoptosis. [47] As the most important cells in the vasculature and the protagonist of angiogenesis, in the early stage of cell therapy intervention for patients with $\mathrm{SCl}$, if the induction of proliferation and migration of vascular endothelial cells can be considered, the secondary damage will be effectively reduced and a microenvironment conducive to nerve repair will be created. And with that in mind, in our study, OECs, an important candidate for SCI repair, and the strategies to promote early-stage-angiogenesis in spinal cord post-injury were combined.

In the present study, for reinforcing the function of OECs, we still use the previous activation processing method. [15] As shown in Fig. 6, In vitro experiments, we observed the indirect and direct effects of OECs on biological behaviors of vascular endothelial cells using OEC-CM and OECs suspensions, respectively. We found that OECs-CM can induce the neovascular sprouting from the broken ends of blood vessels, promote the proliferation and migration of endothelial cells, and form the two-dimensional structure of capillary-like lumen.

In order to better fit the complex situation in vivo, we designed the co-culture-scratch-wound system of OECs and RAECs from the same species, and used the culture environment without adding mitomycin (so that the proliferation and migration factors can be combined), then we found that OECs can promoted endothelial cell proliferation and migration in such a direct contact environment. It is worth emphasizing that both the activated OECs and their CM showed the strongest biological effect in all groups, the findings also complement the potential of OECs activated by LPS\&CCM that we discussed earlier. [14] 
Moreover, according to our results of rat aortic ring assay and previous reports, we set the time for the animals to be sacrificed and spinal-cords-isolated one week after the injury. After confirming the success of the establishment of $\mathrm{SCl}$ model and cell transplantation, we used the methods of immunohistochemistry and micro-CT angiography to analyze the details of spinal angiogenesis: the number of endothelial cells and the number of effective vessels in two and three dimensions, respectively. From the perspective of $\mathrm{CD} 31^{+}$endothelial cells in immunohistochemical results, although there was a certain significant proliferation in AOT groups, there were still a large number of endothelial cells lost in the spinal cord after compressional injury, which have far fewer $\mathrm{CD} 31^{+}$endothelial cells in the experimental group than in the sham group. Surprisingly, micro-CT angiography results showed that the AOT or UOT group and the sham group were very close in terms of effective vessel volume and number. The loss of endothelial cells after tissue injury and the near-normal vascular structure may seem paradoxical, but it is just consistent with the early manifestations of angiogenesis. Our results may reveal that the processes of angiogenesis promoted by OECs were more dependent on endothelial cells migration than their proliferation in the injured area. The migration of native cells from the wound boundary is one of the key steps in the wound healing process, so endothelial cell migration is indeed the most important part of angiogenesis at the background of tissue lesion. [48] This can explain to some extent why there are differences in repair degree of cell (CD31+ cell rate) and tissue (angiography) in our in vivo studies. The amplitude difference between proliferation and migration is worth further discussion and study: previous studies have found that different subtypes of endothelial cells play different roles in new blood vessel formation, with some more responsible for migration guidance and others for proliferation. For instance, endothelial "tip cells" at the forefront of a sprouting vessel navigate by extending filopodia act as the formation-spearhead, mainly responsible for migration and guide; "stalk cells" trail behind and elongate the branch, who can proliferate under the guidance of the tip. [49] They found that the tips of the sprouts were composed of highly migratory cells. in conclusion, tip cells indeed mediate the initiation of sprouting at the broken ends of blood vessels who have strong migration potential rather than proliferative potential. The stalk cells that follow the tip cell build the body of the growing sprout, and after their spatial rearrangement will form a lumen where blood can flow. So, the endothelial cells' migration precedes proliferation chronologically and this process is more pronounced in vivo. Therefore, the determination of proliferation (e.g. CCK-8 array) and migration ability (e.g. wound healing or aortic ring assay) under the premise of endothelial subtype differentiation and time-continuous observations can help define the pattern of angiogenesis in the $\mathrm{SCl}$ condition in detail, which can provide a theoretical basis for the clinical sequential treatment of $\mathrm{SCl}$ to some extent. To specifically explore the progressive relationship between migration and proliferation, followed these conclusions, the detailed structural pattern changes of angiogenesis after $\mathrm{SCl}$ can be the focus of subsequent research.

Our above-mentioned results demonstrated convincingly that OECs, especially activated OECs, can indirectly or directly promote endothelial cell proliferation, migration and then induce neovascularizing. In fact, endothelial cells' migration is an important of the earlier steps in the angiogenic cascade - a group of cells coordinate their movements toward a chemotactic gradient and by establishing a precise hierarchy with leader and follower cells. [50] This pattern was named Connective Cell Migration, and the 
two most important elements of this process are chemokines and leadership cells (tip cells). [51] Hence, combining what we've seen in vivo and in vitro, which chemokines or growth factors promote spinal angiogenesis mediated by activated OECs is the guiding question of our study.

Through the binding reaction of membrane (preloaded growth factors binding target) and OECs-CM, we did screen for high expression of VEGF-A and PDGF-AA in the activated group. Angiogenesis is the process of new blood vessel formation, are critical during development and subsequent physiologic homeostasis, the current academic consensus is that VEGF-A is one of the most important growth factors for stimulation of vascular development and angiogenesis. $[52,53]$ And Platelet-derived growth factor (PDGF-AA) is a potent mitogen and chemoattractant for smooth muscle cells and fibroblasts in culture, which can induce the directed migration and proliferation of arterial smooth muscle cells and fibroblasts. $[54,55]$ Among these participant cell, endothelial cells are the protagonist-cells in the period of new vessel formation who can display the remarkable capability to switch rapidly from a quiescent state to a highly migratory and proliferative state during vessel sprouting. [56] Thus, our investigation of the mechanism used endothelial cell lines as the object of intervention and observation.

The activation of the PI3K/Akt pathway has been proved to stimulate several essential cellular responses intrinsic to angiogenesis such as survival, migration and tube formation in many studies, and one of the important pathways to perform that is the binding of VEGF-A to VEGFR-2 activates the receptor's kinase activity and engages PI3K and its downstream effector Akt. [57-59] Prompted by our array screening results, we wanted to confirm that this pathway is also activated during angiogenesis in injured spinal cord. We verified this pathway by direct observation of kinase phosphorylation levels (Western blotting) and by indirect comparative observation before and after via pathway inhibition (Transwell migration and Edu incorporation assay), and all of these results confirm that the activation of PI3K/Akt pathway is an important mechanism for OECs to promote angiogenesis.

Meanwhile, the classical pathway has multiple effects. Take the PI3K/Akt pathway as an example, from the neuron's perspective, several studies observed that PI3K/Akt signal pathway was critically mediated neuroprotection, axonal regeneration and neurogenesis [60, 61]; from the OEC's perspective, the activation of PI3K/Akt signal pathway regulated by OECs can protect and reinforce itself in the form of feedback, such as promote migration, survival and proliferation [62, 63]; from the perspective of immune microenvironment, this pathway can prevent neural injury by modulating microglia/macrophage polarization and antioxidant effects [64, 65]; and from the perspective of stem cell transplantation, Activation of PI3K/Akt pathway by exogenous intervention can promote differentiation of endogenous neural stem cells and even exogenous stem cells with SCl-treatment potential, such as spermatogonial stem cells [66-68]. Taken together, the mechanisms of OECs promoting the repair of nervous system injury are indeed multifaceted, and our present study just elucidate partial for that. This demonstrates the comprehensive advantages of cell therapy, and our exploration of activation intervention and other potential of OECs is also a theoretical supplement to cell therapy. As we prepared and planned, the core idea of the sequential cell transplantation therapy is to improve the microenvironment with OECs before repair and replenishment of neurons: sufficient angiogenesis and suppressed inflammatory response 
created by transplanted OECs can facilitate the microenvironment at the injured site (Act as a pioneer). On this microenvironment of above mentioned, we speculated that it is conducive to differentiation of exogenous stem cells into neurons, leading to greatly improvement in the efficiency of nerve repair.

\section{Conclusion}

In our study, we observed changes of vascular endothelial cells and vascular tissues after OECs intervention in vivo and in vitro, involving indirect effects (conditioned media for OECs) and direct effects (co-culture in vitro and cell transplantation in vivo), and it was found in protein array screening and mechanism-related experiments that the PI3K/Akt pathway was involved in these processes. Our study further confirmed the pro-angiogenic potential of OECs in treating $\mathrm{SCl}$, and also laid foundation for our sequential therapeutic concept of microenvironment amelioration-stem cell transplantation in some extents.

\section{Abbreviations}

SCI: Spinal cord injury; OECs: Olfactory ensheathing cells; LPS: lipopolysaccharide; CCM: curcumin; BSCB: blood-brain-spinal cord barrier; VEGF: vascular endothelial growth factor; DMEM: Dulbecco's modified Eagle's medium; FBS: fetal bovine serum; PLL: poly-Lysine; DMSO: dimethyl sulfoxide; BSA: bovine serum albumin; EdU: 5-ethynyl-2-deoxyuridine; hUVECs: Human umbilical vein endothelial cells; ECM: endothelial cell medium; ECGs: endothelial cell growth supplement; DAPI: 2-(4-Amidinophenyl)-6indolecarbamidine dihydrochloride; GFP: green fluorescent protein; CM: conditional-media; Act-OECs: Activated-OECs; Unact-OECs: Unactivated-OECs; ACM: Activated-OECs conditional-media; UCM: Unactivated-OECs conditional-media; CCK-8: Cell Counting Kit-8; AOT: activated-OECs transplantation; UOT: unactivated-OECs transplantation group; BBB Locomotor Scale: Basso, Beattie, and Bresnahan Locomotor Scale; HRP: Poly-horseradish peroxidase; micro-CT: micro-computed tomography; PVDF: poly vinylidene fluoride; TBST: Tris-buffered saline-Tween20; IVD: intensity of density value; SD: standard deviation; IGFBP-5: insulin-like growth factor-binding protein-5; PDGF-AA: platelet derived growth factor AA; HGF: hepatic growth factor; GDNF: glial cell line -derived neurotrophic factor; a-NGF: a-nerve growth factor; BDNF: brain-derived neurotrophic factor; PI3K: phosphatidylinositol 3-kinase; Akt: Protein Kinase B.

\section{Declarations}

Ethics approval This study was granted ethical approval from the Ethics Committee of Honghui Hospital, Xi'an Jiaotong University (Number: 2020G28). And we certify that all experimental procedures were performed in accordance with the Guide of Laboratory Animal Care and Use from the United States National Institution of Health and were approved by the Institutional Animal Care and Use Committee (IACUC) of Xi'an Jiaotong University, SN, China.

Consent for publication Not applicable. 
Availability of data and materials The datasets supporting the conclusions of this article are available in the PubMed repository (https://pubmed.ncbi.nlm.nih.gov).

Competing interests The authors declare that they have no competing interests.

Funding This work was funded by the National Natural Science Foundation of China (grants 81772357 and 81371411), a key grant from the National Nature Science Foundation of China (81830077), Natural Science Foundation of Shaanxi Province (2020JM-686) and Xi'an Science and Technology Research Project (2019114913YX004SF037(3)).

Authors' contributions XHW was responsible for designing the protocol, writing the protocol, performing experimental procedures, participating in animals surgery, evaluating and writing the article; $\mathrm{CJ}$ was responsible for detailing the protocol, participate in experimental procedures in each tests, result collection and result analysis; YYZ participating in animals surgery and putting forward the ideas of clinical transformation, ZC guiding the modeling surgery, screening potentially eligible studies; YYZ, ZYW and FT assisting in the operation, recording partial postoperative indicators; JL providing instructions on the use of experimental reagents; $\mathrm{HY}$ and HF improvement our ideas solving some technical problems encountered (Methodology); DJH presiding over the direction of the project and funding acquisition.

Acknowledgements Not applicable.

\section{References}

1. Holmes D. Spinal-cord injury: spurring regrowth. Nature. 2017; 552(7684):S49.

2. Hutson TH, Di Giovanni S. The translational landscape in spinal cord injury: focus on neuroplasticity and regeneration. Nat Rev Neurol. 2019; 15(12):732-45.

3. Zhou P, Guan J, Xu P, Zhao J, Zhang C, Zhang B, Mao Y, Cui W. Cell Therapeutic Strategies for Spinal Cord Injury. Adv Wound Care (New Rochelle). 2019; 8(11):585-605.

4. Gómez RM, Sánchez MY, Portela-Lomba M, Ghotme K, Barreto GE, Sierra J, Moreno-Flores MT. Cell therapy for spinal cord injury with olfactory ensheathing glia cells (OECs). Glia. 2018; 66(7):1267-301.

5. Yang $\mathrm{H}, \mathrm{He} \mathrm{BR}$, Hao DJ. Biological roles of olfactory ensheathing cells in facilitating neural regeneration: a systematic review. Mol Neurobiol. 2015; 51(1):168-79.

6. Vadivelu RK, Ooi CH, Yao RQ, Tello VJ, Pastrana E, Diaz-Nido J, Lim F, Ekberg JA, Nguyen NT, St JJ. Generation of three-dimensional multiple spheroid model of olfactory ensheathing cells using floating liquid marbles. Sci Rep. 2015; 5:15083.

7. Andries L, Van Hove I, Moons L, De Groef L. Matrix Metalloproteinases During Axonal Regeneration, a Multifactorial Role from Start to Finish. Mol Neurobiol. 2017; 54(3):2114-25. 
8. Pastrana E, Moreno-Flores MT, Gurzov EN, Avila J, Wandosell F, Diaz-Nido J. Genes associated with adult axon regeneration promoted by olfactory ensheathing cells: a new role for matrix metalloproteinase 2. J Neurosci. 2006; 26(20):5347-59.

9. Hu R, Zhou J, Luo C, Lin J, Wang X, Li X, Bian X, Li Y, Wan Q, Yu Y, et al. Glial scar and neuroregeneration: histological, functional, and magnetic resonance imaging analysis in chronic spinal cord injury. J Neurosurg Spine. 2010; 13(2):169-80.

10. Vincent AJ, Choi-Lundberg DL, Harris JA, West AK, Chuah MI. Bacteria and PAMPs activate nuclear factor kappaB and Gro production in a subset of olfactory ensheathing cells and astrocytes but not in Schwann cells. Glia. 2007; 55(9):905-16.

11. Wright AA, Todorovic M, Tello-Velasquez J, Rayfield AJ, St JJ, Ekberg JA. Enhancing the Therapeutic Potential of Olfactory Ensheathing Cells in Spinal Cord Repair Using Neurotrophins. Cell Transplant. 2018; 27(6):867-78.

12. Su Z, Chen J, Qiu Y, Yuan Y, Zhu F, Zhu Y, Liu X, Pu Y, He C. Olfactory ensheathing cells: the primary innate immunocytes in the olfactory pathway to engulf apoptotic olfactory nerve debris. Glia. 2013; 61(4):490-503.

13. Su Z, He C. Olfactory ensheathing cells: biology in neural development and regeneration. Prog Neurobiol. 2010; 92(4):517-32.

14. Hao DJ, Liu C, Zhang L, Chen B, Zhang Q, Zhang R, An J, Zhao J, Wu M, Wang Y, et al. Lipopolysaccharide and Curcumin Co-Stimulation Potentiates Olfactory Ensheathing Cell Phagocytosis Via Enhancing Their Activation. Neurotherapeutics. 2017; 14(2):502-18.

15. Guo J, Cao G, Yang G, Zhang Y, Wang Y, Song W, Xu Y, Ma T, Liu R, Zhang Q, et al. Transplantation of activated olfactory ensheathing cells by curcumin strengthens regeneration and recovery of function after spinal cord injury in rats. Cytotherapy. 2020; 22(6):301-12.

16. He BR, Xie ST, Wu MM, Hao DJ, Yang H. Phagocytic removal of neuronal debris by olfactory ensheathing cells enhances neuronal survival and neurite outgrowth via p38MAPK activity. Mol Neurobiol. 2014; 49(3):1501-12.

17. Fan B, Wei Z, Yao X, Shi G, Cheng X, Zhou X, Zhou H, Ning G, Kong X, Feng S. Microenvironment Imbalance of Spinal Cord Injury. Cell Transplant. 2018; 27(6):853-66.

18. Cao Y, Xu Y, Chen C, Xie H, Lu H, Hu J. Local delivery of USC-derived exosomes harboring ANGPTL3 enhances spinal cord functional recovery after injury by promoting angiogenesis. Stem Cell Res Ther. 2021; 12(1):20.

19. Zhong D, Cao Y, Li CJ, Li M, Rong ZJ, Jiang L, Guo Z, Lu HB, Hu JZ. Neural stem cell-derived exosomes facilitate spinal cord functional recovery after injury by promoting angiogenesis. Exp Biol Med 
(Maywood). 2020; 245(1):54-65.

20. Rauch MF, Hynes SR, Bertram J, Redmond A, Robinson R, Williams C, Xu H, Madri JA, Lavik EB. Engineering angiogenesis following spinal cord injury: a coculture of neural progenitor and endothelial cells in a degradable polymer implant leads to an increase in vessel density and formation of the blood-spinal cord barrier. Eur J Neurosci. 2009; 29(1):132-45.

21. Figley SA, Khosravi R, Legasto JM, Tseng YF, Fehlings MG. Characterization of vascular disruption and blood-spinal cord barrier permeability following traumatic spinal cord injury. J Neurotrauma. 2014; 31(6):541-52.

22. Zheng B, Zhou Y, Zhang H, Yang G, Hong Z, Han D, Wang Q, He Z, Liu Y, Wu F, et al. Dl-3-nbutylphthalide prevents the disruption of blood-spinal cord barrier via inhibiting endoplasmic reticulum stress following spinal cord injury. Int J Biol Sci. 2017; 13(12):1520-31.

23. Lee JY, Choi HY, Park CS, Ju BG, Yune TY. Mithramycin A Improves Functional Recovery by Inhibiting BSCB Disruption and Hemorrhage after Spinal Cord Injury. J Neurotrauma. 2018; 35(3):508-20.

24. Qi F, Wang Y, Ma T, Zhu S, Zeng W, Hu X, Liu Z, Huang J, Luo Z. Electrical regulation of olfactory ensheathing cells using conductive polypyrrole/chitosan polymers. Biomaterials. 2013; 34(7):1799-1809.

25. Gomes ED, Mendes SS, Assunção-Silva RC, Teixeira FG, Pires AO, Anjo SI, Manadas B, Leite-Almeida H, Gimble JM, Sousa N, et al. Co-Transplantation of Adipose Tissue-Derived Stromal Cells and Olfactory Ensheathing Cells for Spinal Cord Injury Repair. Stem Cells. 2018; 36(5):696-708.

26. López-Vales R, García-Alías G, Forés J, Navarro X, Verdú E. Increased expression of cyclo-oxygenase 2 and vascular endothelial growth factor in lesioned spinal cord by transplanted olfactory ensheathing cells. J Neurotrauma. 2004; 21(8):1031-43.

27. Richter MW, Fletcher PA, Liu J, Tetzlaff W, Roskams AJ. Lamina propria and olfactory bulb ensheathing cells exhibit differential integration and migration and promote differential axon sprouting in the lesioned spinal cord. J Neurosci. 2005; 25(46):10700-11.

28. Ramer LM, Au E, Richter MW, Liu J, Tetzlaff W, Roskams AJ. Peripheral olfactory ensheathing cells reduce scar and cavity formation and promote regeneration after spinal cord injury. J Comp Neurol. 2004; 473(1):1-15.

29. Nash HH, Borke RC, Anders JJ. New method of purification for establishing primary cultures of ensheathing cells from the adult olfactory bulb. Glia. 2001;34(2):81-7.

30. Yue PY, Wong DY, Wu PK, Leung PY, Mak NK, Yeung HW, Liu L, Cai Z, Jiang ZH, Fan TP, et al. The angiosuppressive effects of 20(R)- ginsenoside Rg3. Biochem Pharmacol. 2006; 72(4):437-45. 
31. Wang T, Fang X, Yin ZS. Endothelial progenitor cell-conditioned medium promotes angiogenesis and is neuroprotective after spinal cord injury. Neural Regen Res. 2018; 13(5):887-95.

32. Yi M, Wu Y, Long J, Liu F, Liu Z, Zhang YH, Sun XP, Fan ZX, Gao J, Si J, et al. Exosomes secreted from osteocalcin-overexpressing endothelial progenitor cells promote endothelial cell angiogenesis. Am J Physiol Cell Physiol. 2019; 317(5):C932-41.

33. Vaughn CN, lafrate JL, Henley JB, Stevenson EK, Shlifer IG, Jones TB. Cellular neuroinflammation in a lateral forceps compression model of spinal cord injury. Anat Rec (Hoboken). 2013; 296(8):1229-46.

34. Mcdonough A, Monterrubio A, Ariza J, Martínez-Cerdeño V. Calibrated forceps model of spinal cord compression injury. J Vis Exp. 2015(98).

35. Basso DM, Beattie MS, Bresnahan JC. A sensitive and reliable locomotor rating scale for open field testing in rats. J Neurotrauma. 1995; 12(1):1-21.

36. Yang H, Ling W, Vitale A, Olivera C, Min Y, You S. ErbB2 activation contributes to de-differentiation of astrocytes into radial glial cells following induction of scratch-insulted astrocyte conditioned medium. Neurochem Int. 2011; 59(7):1010-8.

37. Yao Y, Sun W, Sun Q, Jing B, Liu S, Liu X, Shen G, Chen R, Wang H. Platelet-Derived Exosomal MicroRNA-25-3p Inhibits Coronary Vascular Endothelial Cell Inflammation Through Adam10 via the NFKB Signaling Pathway in ApoE(-/-) Mice. Front Immunol. 2019; 10:2205.

38. Wang CQ, Lin CY, Huang YL, Wang SW, Wang Y, Huang BF, Lai YW, Weng SL, Fong YC, Tang CH, et al. Sphingosine-1-phosphate promotes PDGF-dependent endothelial progenitor cell angiogenesis in human chondrosarcoma cells. Aging (Albany NY). 2019; 11(23):11040-53.

39. Ferrara N, Gerber HP, Lecouter J. The biology of VEGF and its receptors. Nat Med. 2003; 9(6):669-76.

40. Zheng Z, Zeng Y, Zhu X, Tan Y, Li Y, Li Q, Yi G. ApoM-S1P Modulates Ox-LDL-Induced Inflammation Through the PI3K/Akt Signaling Pathway in HUVECs. Inflammation. 2019; 42(2):606-17.

41. Zhu GS, Tang LY, Lv DL, Jiang M. Total Flavones of Abelmoschus manihot Exhibits Pro-Angiogenic Activity by Activating the VEGF-A/VEGFR2-PI3K/Akt Signaling Axis. Am J Chin Med. 2018; 46(3):567-83.

42. Zhang X, Zhao F, Zhao JF, Fu HY, Huang XJ, Lv BD. PDGF-mediated PI3K/AKT/ $\beta$-catenin signaling regulates gap junctions in corpus cavernosum smooth muscle cells. Exp Cell Res. 2018; 362(2):252-9.

43. Ahuja CS, Wilson JR, Nori S, Kotter M, Druschel C, Curt A, Fehlings MG. Traumatic spinal cord injury. Nat Rev Dis Primers. 2017; 3:17018.

44. Fan B, Wei Z, Yao X, Shi G, Cheng X, Zhou X, Zhou H, Ning G, Kong X, Feng S. Microenvironment Imbalance of Spinal Cord Injury. Cell Transplant. 2018; 27(6):853-66. 
45. Oudega M. Molecular and cellular mechanisms underlying the role of blood vessels in spinal cord injury and repair. Cell Tissue Res. 2012; 349(1):269-88.

46. Kamei N, Kwon SM, Alev C, Nakanishi K, Yamada K, Masuda H, Ishikawa M, Kawamoto A, Ochi M, Asahara T. Ex-vivo expanded human blood-derived CD133+ cells promote repair of injured spinal cord. J Neurol Sci. 2013; 328(1-2):41-50.

47. Casella GT, Bunge MB, Wood PM. Endothelial cell loss is not a major cause of neuronal and glial cell death following contusion injury of the spinal cord. Exp Neurol. 2006; 202(1):8-20.

48. Velnar T, Bailey T, Smrkolj V. The wound healing process: an overview of the cellular and molecular mechanisms. J Int Med Res. 2009; 37(5):1528-42.

49. Gerhardt $\mathrm{H}$, Golding $\mathrm{M}$, Fruttiger $\mathrm{M}$, Ruhrberg $\mathrm{C}$, Lundkvist A, Abramsson A, Jeltsch M, Mitchell C, Alitalo $\mathrm{K}$, Shima D, et al. VEGF guides angiogenic sprouting utilizing endothelial tip cell filopodia. J Cell Biol. 2003; 161(6):1163-77.

50. Nowak-Sliwinska P, Alitalo K, Allen E, Anisimov A, Aplin AC, Auerbach R, Augustin HG, Bates DO, van Beijnum JR, Bender R, et al. Consensus guidelines for the use and interpretation of angiogenesis assays. Angiogenesis. 2018; 21(3):425-532.

51. Haeger A, Wolf K, Zegers MM, Friedl P. Collective cell migration: guidance principles and hierarchies. Trends Cell Biol. 2015; 25(9):556-66.

52. Apte RS, Chen DS, Ferrara N. VEGF in Signaling and Disease: Beyond Discovery and Development. Cell. 2019; 176(6):1248-64.

53. Hu K, Olsen BR. The roles of vascular endothelial growth factor in bone repair and regeneration. Bone. 2016; 91:30-8.

54. Hosang M, Rouge M. Human vascular smooth muscle cells have at least two distinct PDGF receptors and can secrete PDGF-AA. J Cardiovasc Pharmacol. 1989; 14 Suppl 6:S22-6.

55. Bornfeldt KE, Raines EW, Graves LM, Skinner MP, Krebs EG, Ross R. Platelet-derived growth factor. Distinct signal transduction pathways associated with migration versus proliferation. Ann N Y Acad Sci. 1995; 766:416-30.

56. Eelen G, de Zeeuw P, Simons M, Carmeliet P. Endothelial cell metabolism in normal and diseased vasculature. Circ Res. 2015; 116(7):1231-44.

57. Ruan GX, Kazlauskas A. Axl is essential for VEGF-A-dependent activation of PI3K/Akt. Embo J. 2012; 31(7):1692-1703. 
58. Ferrara N, Gerber HP, Lecouter J. The biology of VEGF and its receptors. Nat Med. 2003; 9(6):669676.

59. Yu M, Liu W, Li J, Lu J, Lu H, Jia W, Liu F. Exosomes derived from atorvastatin-pretreated MSC accelerate diabetic wound repair by enhancing angiogenesis via AKT/eNOS pathway. Stem Cell Res Ther. 2020; 11(1):350.

60. He J, Zhang N, Zhu Y, Jin R, Wu F. MSC spheroids-loaded collagen hydrogels simultaneously promote neuronal differentiation and suppress inflammatory reaction through PI3K-Akt signaling pathway. Biomaterials. 2021; 265:120448.

61. Hervera A, De Virgiliis F, Palmisano I, Zhou L, Tantardini E, Kong G, Hutson T, Danzi MC, Perry RB, Santos $C$, et al. Reactive oxygen species regulate axonal regeneration through the release of exosomal NADPH oxidase 2 complexes into injured axons. Nat Cell Biol. 2018; 20(3):307-19.

62. Tang YY, Guo WX, Lu ZF, Cheng MH, Shen YX, Zhang YZ. Ginsenoside Rg1 Promotes the Migration of Olfactory Ensheathing Cells via the PI3K/Akt Pathway to Repair Rat Spinal Cord Injury. Biol Pharm Bull. 2017; 40(10):1630-7.

63. Wang YH, Li YC, Huo SJ, Yin ZQ. Alpha-crystallin promotes rat olfactory ensheathing cells survival and proliferation through regulation of PI3K/Akt/mTOR signaling pathways. Neurosci Lett. 2012; 531(2):170-175.

64. Wang G, Shi Y, Jiang X, Leak RK, Hu X, Wu Y, Pu H, Li WW, Tang B, Wang Y, et al. HDAC inhibition prevents white matter injury by modulating microglia/macrophage polarization through the GSK3ß/PTEN/Akt axis. Proc Natl Acad Sci U S A. 2015; 112(9):2853-8.

65. Dong L, Li R, Li D, Wang B, Lu Y, Li P, Yu F, Jin Y, Ni X, Wu Y, et al. FGF10 Enhances Peripheral Nerve Regeneration via the Preactivation of the PI3K/Akt Signaling-Mediated Antioxidant Response. Front Pharmacol. 2019; 10:1224.

66. Yang H, Liu Y, Hai Y, Guo Y, Yang S, Li Z, Gao WQ, He Z. Efficient Conversion of Spermatogonial Stem Cells to Phenotypic and Functional Dopaminergic Neurons via the PI3K/Akt and P21/Smurf2/Nolz1 Pathway. Mol Neurobiol. 2015; 52(3):1654-69.

67. Huang F, Gao T, Wang W, Wang L, Xie Y, Tai C, Liu S, Cui Y, Wang B. Engineered basic fibroblast growth factor-overexpressing human umbilical cord-derived mesenchymal stem cells improve the proliferation and neuronal differentiation of endogenous neural stem cells and functional recovery of spinal cord injury by activating the PI3K-Akt-GSK-3 $\beta$ signaling pathway. Stem Cell Res Ther. 2021; 12(1):468.

68. Yang H, Liu C, Chen B, An J, Zhang R, Zhang Q, Zhao J, He B, Hao DJ. Efficient Generation of Functionally Active Spinal Cord Neurons from Spermatogonial Stem Cells. Mol Neurobiol. 2017; 


\section{Figures}
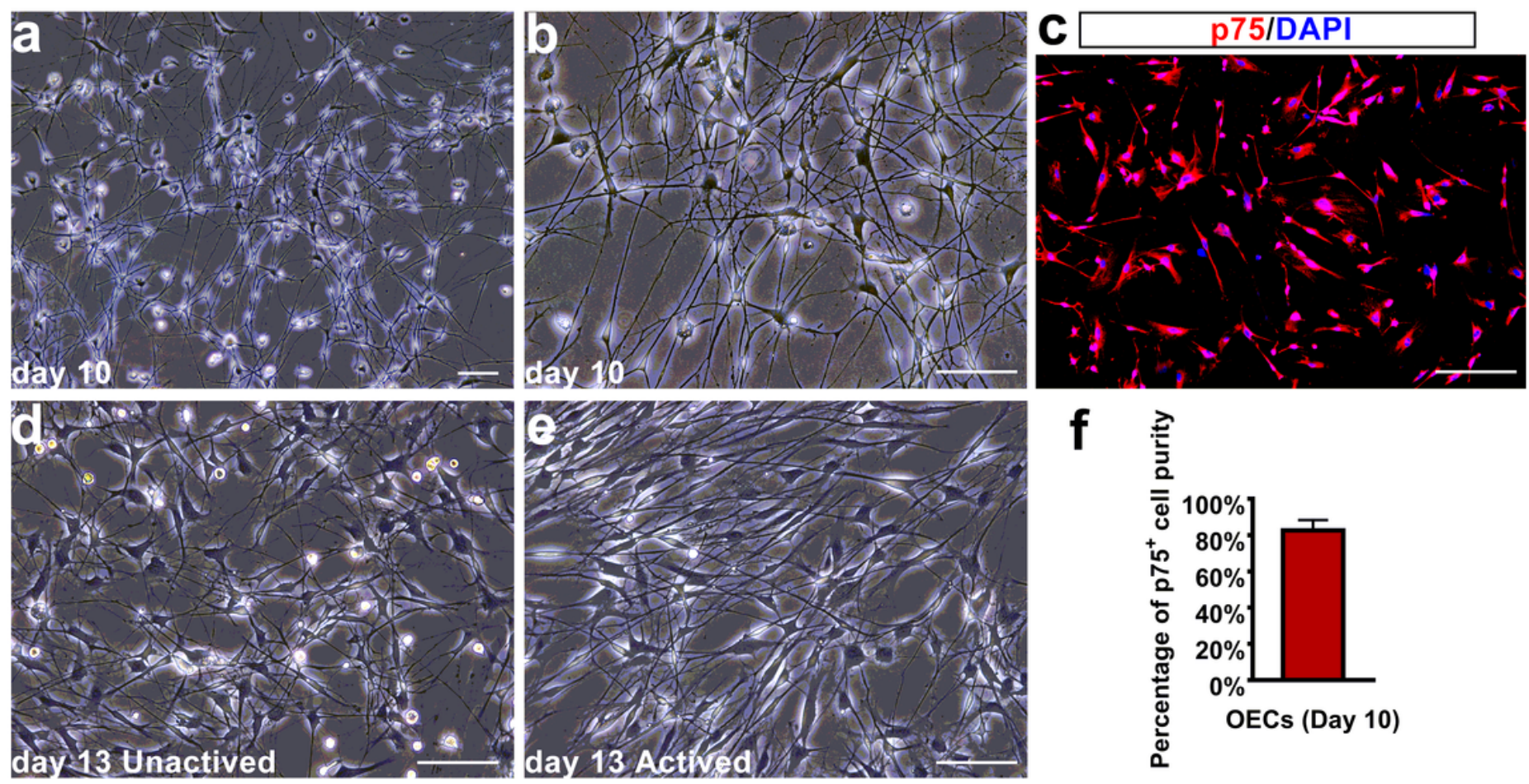

Figure 1

Characterization and identification of OECs. a, b Primary cultured OECs on day 10 under phase contrast microscopes. $\mathbf{c}, \mathbf{f}$ Identification of immunocytochemistry for $\mathrm{p} 75$ and p75 positive cell purity of primary cultured OECs. d, e Primary cultured OECs on day 13 (three days after subculture) with or without activation under $20 \times$ contrast microscopes. Scale bar: $100 \mu \mathrm{m}$. 


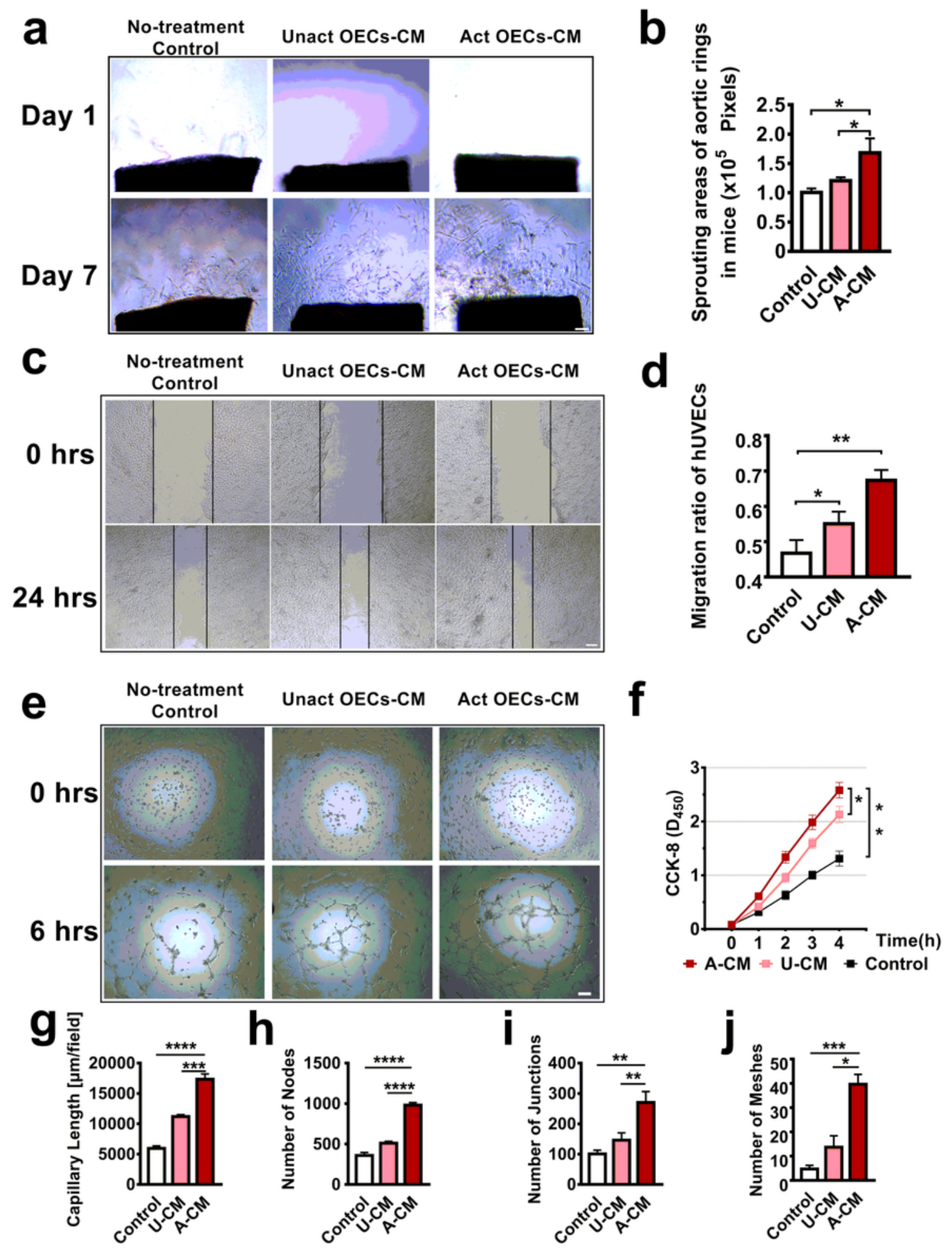

Figure 2

Effect of activated OECs-CM on biological behaviors of vascular endothelial cells. a In vitro representative photomicrograph of micro-vessels sprouted from young rat aortic rings embedded in Matrigel and incubated with OECs-CM or control media. Scale bar: $100 \mu \mathrm{m}$. b Quantitative analysis of sprout areas induced by incubation with each media group. c Phase-contrast micrographs of HUVECs at initial time and $24 \mathrm{~h}$ following monolayer scratch wounding. Scale bar: $100 \mu \mathrm{m}$. d The migration of HUVECs within 
the scratch in the presence of different media groups. Healing index = (initial area - final area) $/$ initial area $\times 100 \%$. e Representative images of HUVEC tube formation in vitro after culturing with OECs-CM or control media. Scale bar: $100 \mu \mathrm{m}$. f Effects of OECs-CM culture on cell proliferation by CCK-8 in HUVECs. g-j Quantitative evaluation of the number of capillary lengths, number of nodes, number of junctions (branch points) and number of meshes(loops) after treating HUVECs with OECs-CM. All data are reported as the mean \pm SD of results from three independent experiments. ACM: activated OECs-CM, UCM: unactivated OECs-CM, $\mathrm{n}=3 ; * P<0.05, * \star P<0.01,{ }^{*} * * P<0.005,{ }^{*} * \star * P<0.001$ vs the corresponding groups.
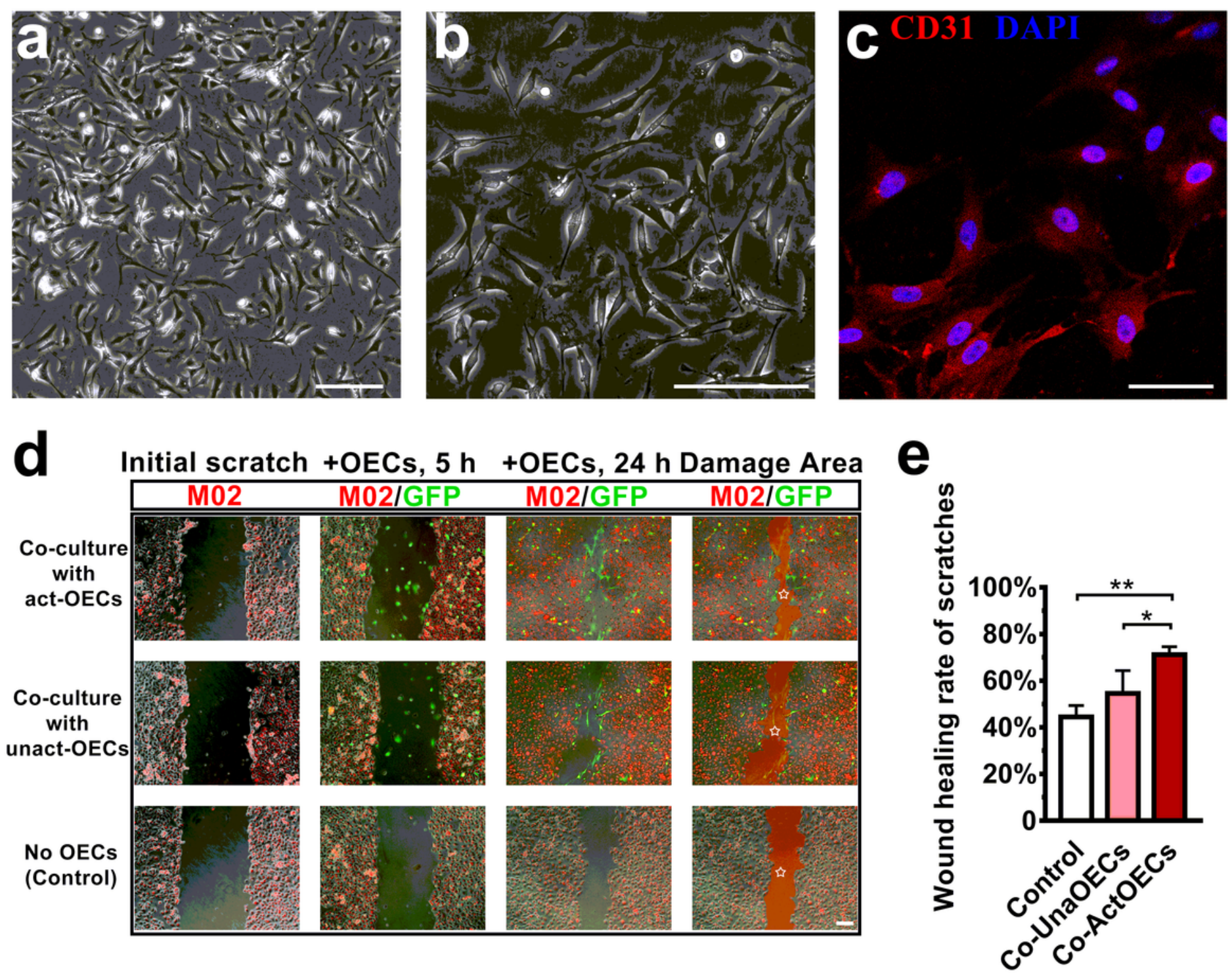

Figure 3

Effect of activated OECs on healing of RAECs wound scratches by co-culture method in vitro. $\mathbf{a}, \mathbf{b}$ Characterization of RAECs primary cultured on day 9 under the phase contrast microscopes. Scale bar: $100 \mu \mathrm{m}$. c Identification of immunofluorescence for CD31 of RAECs. Scale bar: $50 \mu \mathrm{m}$. d Simulated wound scratches of RAECs labeled with red fluorescent probe M02 (Initial stretches column) and co- 
cultured with OECs from GFP transgenic rats at different time point after OECs inoculation, and the final scratch areas were covered with translucent red and marked by stars. Scale bar: $100 \mu \mathrm{m}$. e The wound healing rate for the three groups shown in $\mathbf{d}$. Scale bar: $100 \mu \mathrm{m}$. The data are presented as the means \pm $\mathrm{SD} ; \mathrm{n}=3$ per group. ${ }^{*} P<0.05,{ }^{*} P<0.01$ vs the corresponding groups.
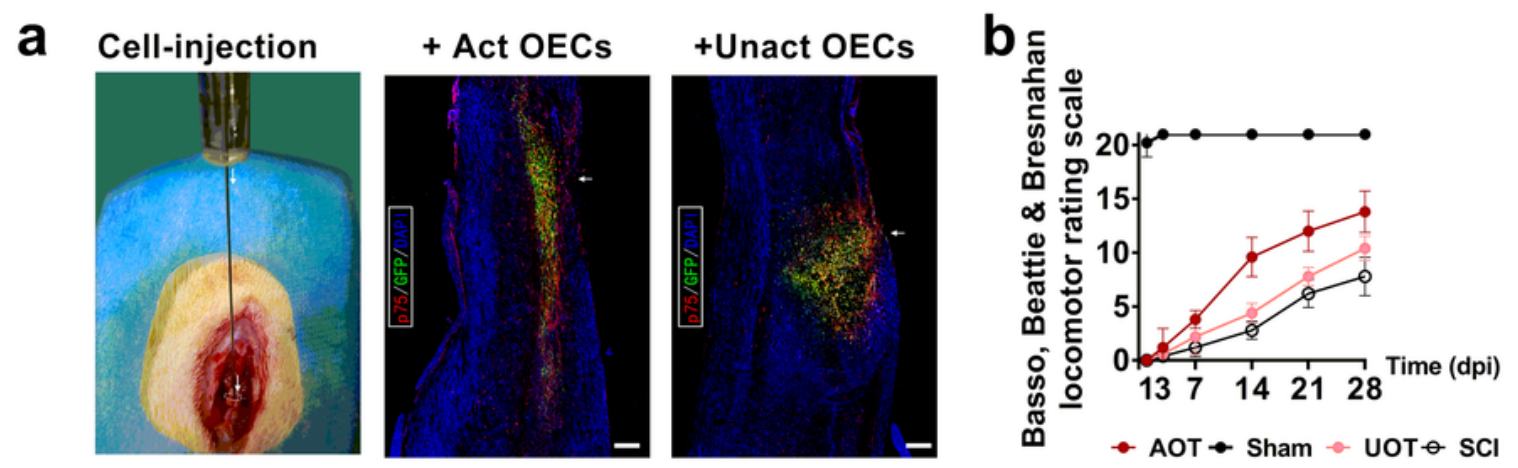

C
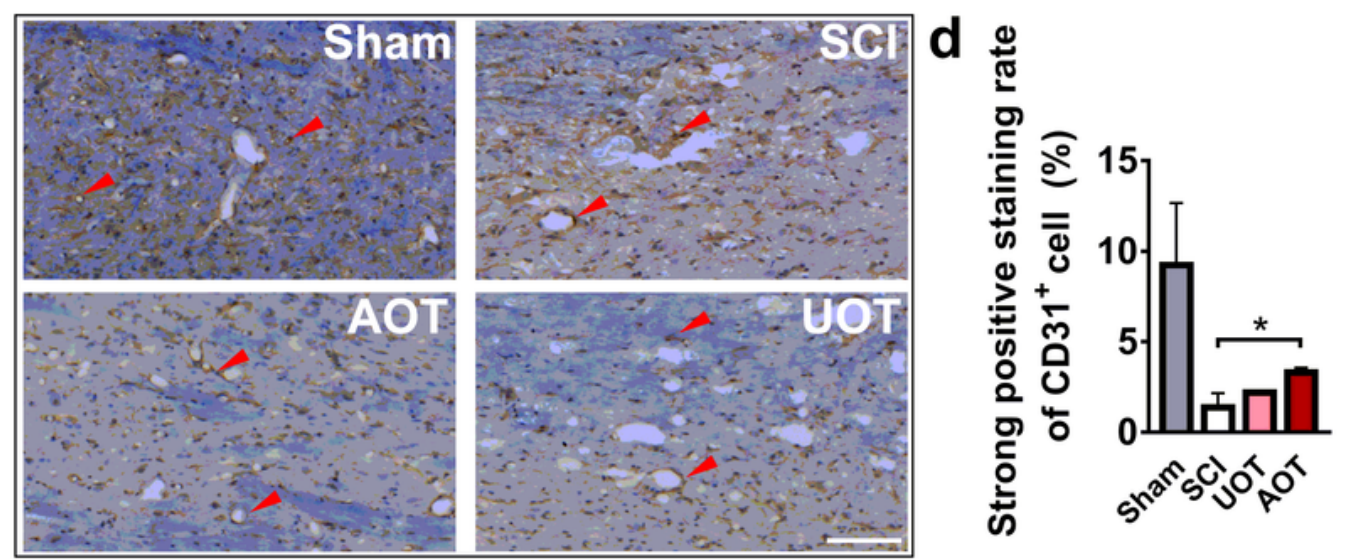

e Sham-operated Spinal cord injury Unact-OECs Trans Act-OECs Trans
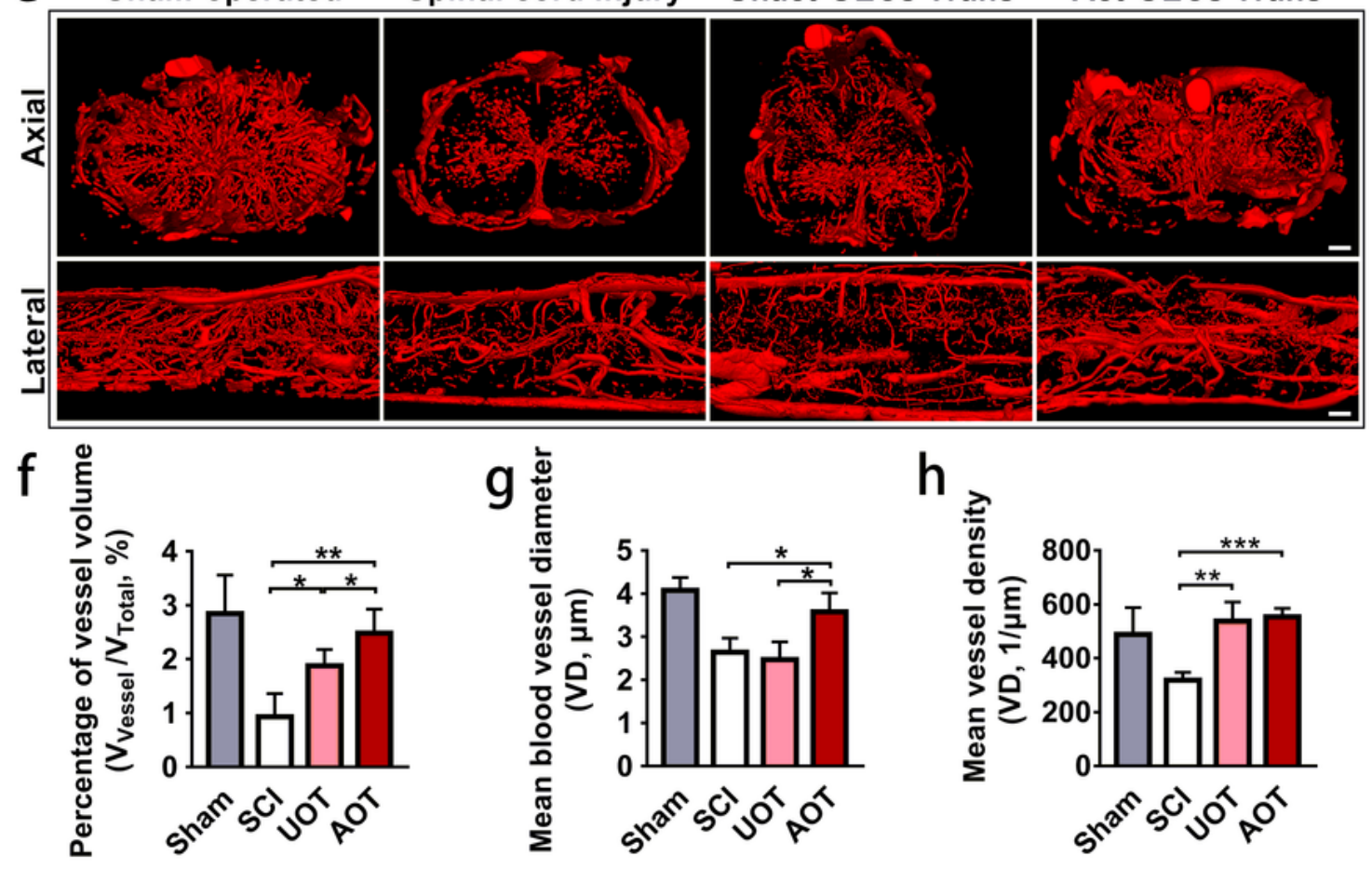

Figure 4 
Effect of OECs on angiogenesis after SCl in vivo. a Injection of OECs from GFP transgenic rats into the spinal cord of rats, and immunofluorescent identification for p75 after one week. they display that OECs all successfully transplanted to the injured areas and the activated OECs have a longer migration area. Scale bar: $200 \mu \mathrm{m}$. b BBB scores within the different observation periods in distinct treated rats after SCl. ( $n=5$ per group) $\mathbf{c}$ Representative images of immunochemical stained sections showing lumens surrounded by positive CD31 cells in the various groups (in vivo). The typical CD31 positive cells are marked by red arrows. $n=3$ per group, scale bars: $100 \mu \mathrm{m}$. $\mathbf{d}$ Positive staining rate of $\mathrm{CD} 31^{+}$cells. AOT: activated OECs transplantation group; UOT: unactivated OECs transplantation group. e Angiographic 3D reconstruction from Micro-CT photographs of spinal cords of rats one week after $\mathrm{SCl}, \mathrm{n}=3$ per group. Scale bar: $200 \mu \mathrm{m}$. $\mathbf{f}-\mathbf{h}$ The percentage of vessel volume (f), mean blood vessel diameter (g) and mean vessel density $(\mathbf{h})$ calculated for the three groups shown in $\mathbf{d}$. All data are reported as the mean \pm SD of results. ${ }^{*} P<0.05,{ }^{* \star} P<0.01,{ }^{* *} P<0.005$ vs the corresponding groups. 
a
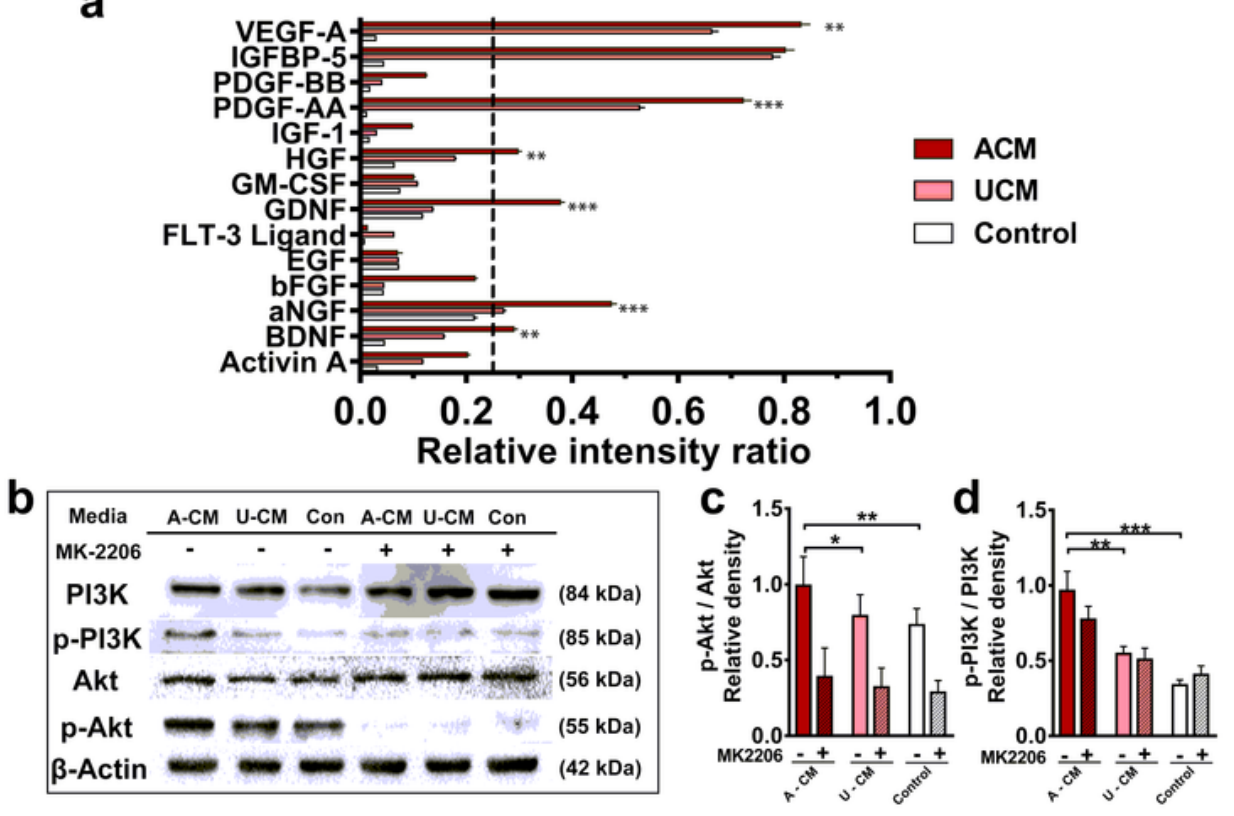

e

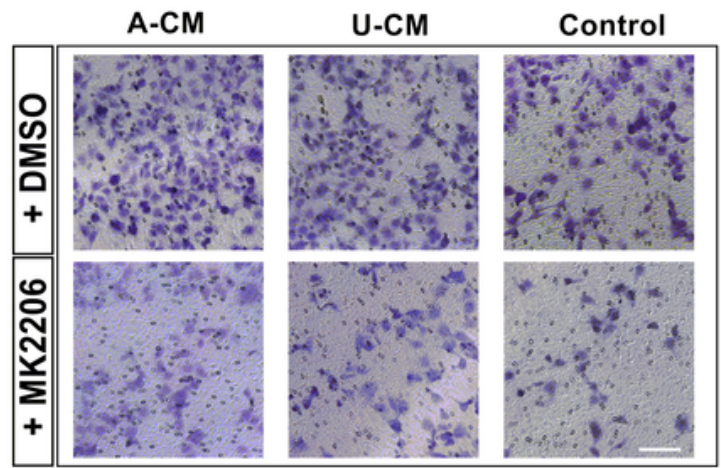

g

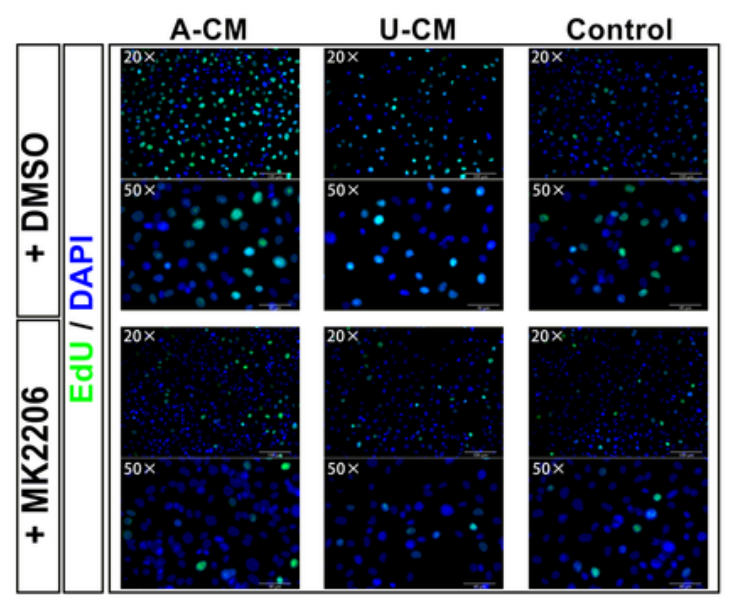

h
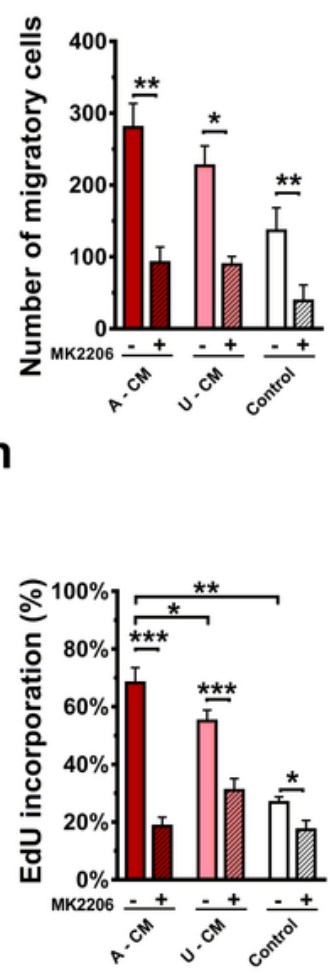

Figure 5

Possible molecular mechanism of the pro-angiogenic effects of Activated OECs. a Growth factor array for angiogenic factors. Each bar shows the relative intensity ratios of growth factors found in activated OECs-CM, unactivated OEC-CM or control media, respectively. b Western blotting detected protein levels of $\mathrm{PI} 3 \mathrm{~K}, \mathrm{p}-\mathrm{PI} \mathrm{KK}, \mathrm{Akt}$, and p-Akt in each group. $\mathbf{c}$ and $\mathbf{d}$ represent the relative density of phosphorylation level of Akt and PI3K, respectively. e, f OECs-CM and MK2206 effect on migration of HUVECs using Transwell 
migration chambers. Scale bar: $100 \mu \mathrm{m} . \mathbf{g}$, h OECs-CM and MK2206 effect on proliferation of HUVECs using EdU incorporation assay. ACM: activated OECs-CM, UCM: unactivated OECs-CM. All values shown are the means $\pm \operatorname{SD}(\mathrm{n}=3) .{ }^{\star} P<0.05,{ }^{*} P P<0.01,{ }^{\star} * * P<0.005 v s$ the corresponding groups.

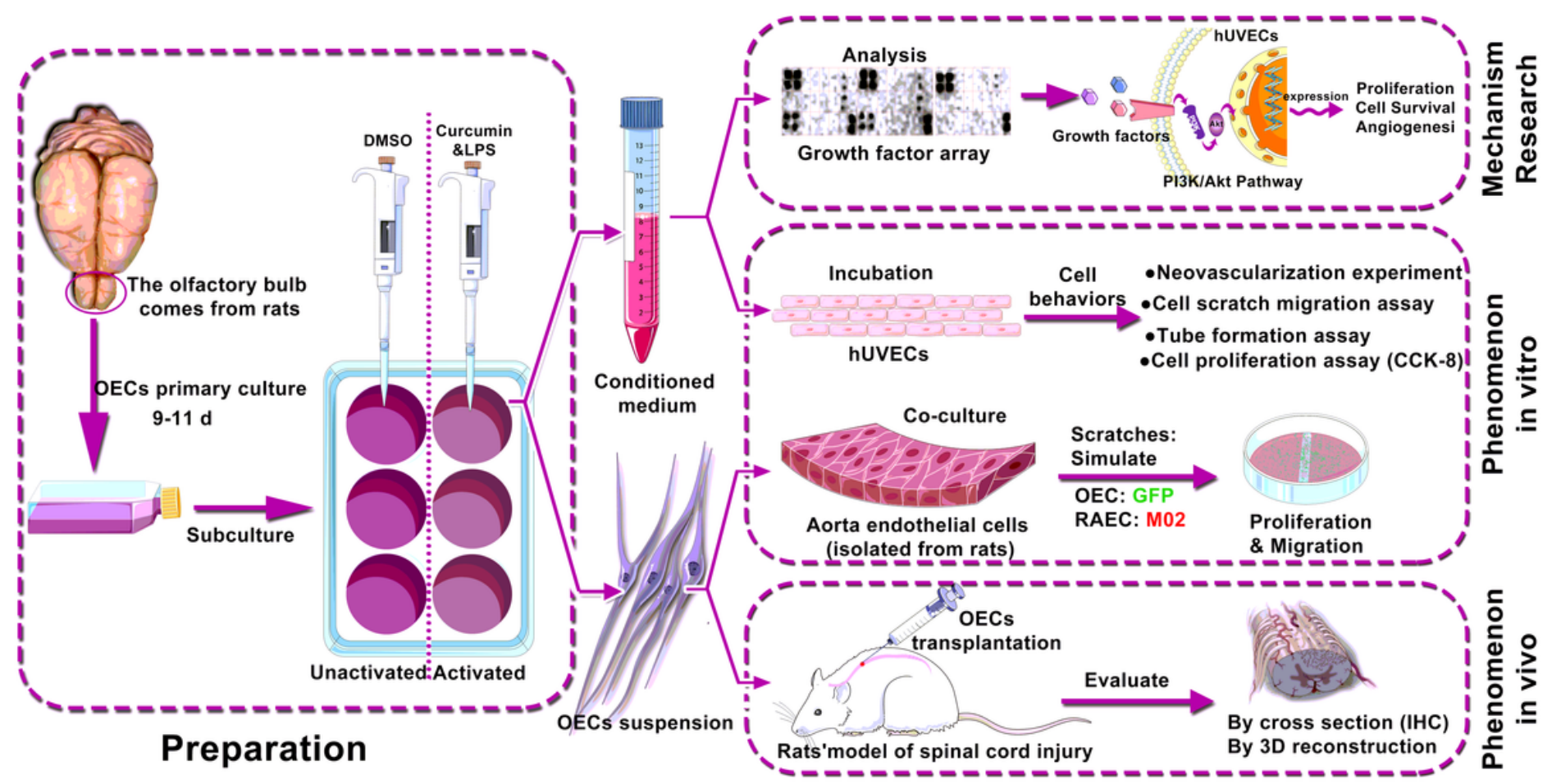

Figure 6

Schematic diagram showing the overall steps of our experiment and the promoting effects of activated OECs on angiogenesis after SCl through the PI3K/Akt pathway. The effects were observed in vitro and in vivo using $\mathrm{CM}$ and cell suspension as interveners separately. In addition, the mechanism behind these effects is revealed to a certain extent under the inspiration from some growth factors screened out by growth factor arrays.

\section{Supplementary Files}

This is a list of supplementary files associated with this preprint. Click to download.

- figures1.jpg

- figures2.jpg

- figures3.jpg

- figures4.jpg

- figures5.jpg

- figures6.jpg 